\title{
New characterizations of multivariate Max-domain of attraction and D-Norms
}

\section{Michael Falk ${ }^{1}$ - Timo Fuller ${ }^{1}$}

Received: 21 July 2020 / Revised: 8 January 2021 / Accepted: 22 March 2021 /

Published online: 5 May 2021

(C) The Author(s) 2021

\begin{abstract}
In this paper we derive new results on multivariate extremes and $D$-norms. In particular we establish new characterizations of the multivariate max-domain of attraction property. The limit distribution of certain multivariate exceedances above high thresholds is derived, and the distribution of that generator of a $D$-norm on $\mathbb{R}^{d}$, whose components sum up to $d$, is obtained. Finally we introduce exchangeable $D$-norms and show that the set of exchangeable $D$-norms is a simplex.
\end{abstract}

Keywords Multivariate extreme value theory - Multivariate max-domain of attraction · $D$-norm · Generator of $D$-norm · Multivariate exceedance .

Co-extremality coefficient · Exchangeable $D$-norms · Extremal exchangeable D-norms

AMS 2000 Subject Classifications $60 \mathrm{G} 70 \cdot 60 \mathrm{E} 99$

\section{Introduction}

Multivariate extreme value theory (MEVT) is the appropriate toolbox for analyzing several extremal events simultaneously. However, MEVT is by no means easy to access; its key results are formulated in a measure theoretic setup in which a common thread is not visible.

Writing the 'angular measure' in MEVT in terms of a random vector, however, provides the missing common thread: Every result in MEVT, every relevant probability distribution, be it a max-stable one or a generalized Pareto distribution, every

Michael Falk

michael.falk@uni-wuerzburg.de

Timo Fuller

timo.fuller@uni-wuerzburg.de

1 University of Wuerzburg, Wuerzburg, Germany 
relevant copula, every tail dependence coefficient etc. can be formulated using a particular kind of norm on multivariate Euclidean space, called a $D$-norm. Deep results like Takahashi's characterizations of multivariate max-stable distributions with independent or completely dependent margins (Takahashi 1987; 1988) turn out to be elementary and easily seen properties of $D$-norms. The letter $D$ means dependence, because a $D$-norm describes the dependence among the margins of a multivariate max-stable distribution, see Theorem 4.1.

Norms are introduced in each introductory course on mathematics as soon as the multivariate Euclidean space is introduced. The definition of an arbitrary $D$-norm requires only the additional knowledge of random variables and their expectations. But $D$-norms do not only constitute the common thread through MEVT; they are of mathematical interest of their own.

$D$-norms were first mentioned in Falk et al. (2004), equation (4.25), and more elaborated in Falk et al. (2011), Section 4.4. But it was recognized only later that $D$-norms are actually the skeleton of MEVT and that they simultaneously provide a mathematical topic, which can be studied independently. The monograph Falk (2019) compiles the contemporary knowledge about $D$-norms and provides an introductory tour through the essentials of MEVT. But $D$-norms can also be seen from a functional analysis perspective as in Ressel (2013), which is Section 1.11 in Falk (2019), or from a stochastic geometry point of view as in Molchanov (2008), presented in detail in Section 1.12 in Falk (2019).

In this paper we establish new results on MEVT and $D$-norms. In Section 2 we recall the definition of $D$-norms and list several basic facts. In Section 3 we specify functions $h:[0, \infty)^{d} \rightarrow[0, \infty)$, such that the number $E(h(\boldsymbol{Z}))$ is generator invariant, i.e., $E(h(\boldsymbol{Z}))$ depends only on the underlying $D$-norm generated by $\boldsymbol{Z}$, but not on the particular generator $\boldsymbol{Z}$. The dual $D$-norm function is a prominent example. But this result also entails the definition of the co-extremality coefficient, which is a measure of pairwise tail dependence of a multivariate distribution. The corresponding matrix of co-extremality coefficients turns out to be positive semidefinite.

In Section 4 we link $D$-norms with MEVT and establish particularly in Theorem 4.4 a new characterization of max-domain of attraction of a random vector $\boldsymbol{X}=\left(X_{1}, \ldots, X_{d}\right)$. An immediate consequence, in Corollary 4.6, is for example the fact that the probability of the event $\sum_{i=1}^{d} X_{i}>t$ is approximately $d / t$ as $t$ increases, independent of the dependence structure among $X_{1}, \ldots, X_{d}$; this was already observed in Barbe et al. (2006).

In Section 5 we derive the limit distribution of certain multivariate exceedances above high thresholds. These results are used in Section 6 to derive among others the distribution of that generator of a $D$-norm on $\mathbb{R}^{d}$, whose components sum up to $d$.

It turns out that Mai and Scherer (2020), which is on exchangeable extreme-value copulas, is actually a contribution to the theory of $D$-norms, as shown in Section 7. To the best of our knowledge, the concept of an exchangeable $D$-norm is introduced here for the first time, and we prove new results for this subfamily of $D$-norms. 


\section{D-Norms}

The following result introduces $D$-norms. For a proof we refer to Lemma 1.1.3 in Falk (2019).

Lemma 2.1 (D-Norms) Let $\boldsymbol{Z}=\left(Z_{1}, \ldots, Z_{d}\right)$ be a random vector, whose components satisfy

$$
Z_{i} \geq 0, \quad E\left(Z_{i}\right)=1, \quad 1 \leq i \leq d
$$

Then

$$
\|\boldsymbol{x}\|_{D}:=E\left(\max _{1 \leq i \leq d}\left(\left|x_{i}\right| Z_{i}\right)\right), \quad \boldsymbol{x} \in \mathbb{R}^{d},
$$

defines a norm, called a D-norm, and $\boldsymbol{Z}$ is called a generator of this D-norm $\|\cdot\|_{D}$.

Denote by $\boldsymbol{e}_{j}=(0, \ldots, 0,1,0, \ldots, 0) \in \mathbb{R}^{d}$ the $j$-th unit vector in $\mathbb{R}^{d}, 1 \leq j \leq d$. Each $D$-norm satisfies

$$
\left\|\boldsymbol{e}_{j}\right\|_{D}=E\left(\max _{1 \leq i \leq d}\left(\delta_{i j} Z_{i}\right)\right)=E\left(Z_{j}\right)=1,
$$

where $\delta_{i j}=1$ if $i=j$ and zero elsewhere, i.e., each $D$-norm is standardized.

Each $D$-norm is monotone as well, i.e., for $\mathbf{0} \leq \boldsymbol{x} \leq \boldsymbol{y}$, where this inequality is taken componentwise, we have

$$
\|\boldsymbol{x}\|_{D}=E\left(\max _{1 \leq i \leq d}\left(x_{i} Z_{i}\right)\right) \leq E\left(\max _{1 \leq i \leq d}\left(y_{i} Z_{i}\right)\right)=\|\boldsymbol{y}\|_{D} .
$$

Example 2.2 Here is a list of $D$-norms and their generators, see Section 1.2 in Falk (2019):

- $\|\boldsymbol{x}\|_{\infty}=\max _{1 \leq i \leq d}\left|x_{i}\right|$, generated by $\boldsymbol{Z}=(1, \ldots, 1)$.

- $\|\boldsymbol{x}\|_{1}=\sum_{i=1}^{d}\left|x_{i}\right|$, generated by $\boldsymbol{Z}=$ random permutation of $(d, 0, \ldots, 0) \in \mathbb{R}^{d}$ with equal probability $1 / d$.

- $\|x\|_{\lambda}=\left(\sum_{i=1}^{d}\left|x_{i}\right|^{\lambda}\right)^{1 / \lambda}, 1<\lambda<\infty$. Let $X_{1}, \ldots, X_{d}$ be independent and identically Fréchet-distributed random variables, i.e., $P\left(X_{i} \leq x\right)=\exp \left(-x^{-\lambda}\right)$, $x>0, \lambda>1$. Then $\boldsymbol{Z}=\left(Z_{1}, \ldots, Z_{d}\right)$ with

$$
Z_{i}:=\frac{X_{i}}{\Gamma\left(1-\frac{1}{\lambda}\right)}, \quad i=1, \ldots, d,
$$

generates $\|\cdot\|_{\lambda}$.

- Let the random vector $\boldsymbol{X}=\left(X_{1}, \ldots, X_{d}\right)$ follow a multivariate normal distribution with mean vector zero, i.e., $E\left(X_{i}\right)=0,1 \leq i \leq d$, and covariance matrix 
$\Sigma=\left(\sigma_{i j}\right)_{1 \leq i, j \leq d}=\left(E\left(X_{i} X_{j}\right)\right)_{1 \leq i, j \leq d}$. Then $\exp \left(X_{i}\right)$ follows a log-normal distribution with mean $\exp \left(\sigma_{i i} / 2\right), 1 \leq i \leq d$, and thus,

$$
\boldsymbol{Z}=\left(Z_{1}, \ldots, Z_{d}\right):=\left(\exp \left(X_{1}-\frac{\sigma_{11}}{2}\right), \ldots, \exp \left(X_{d}-\frac{\sigma_{d d}}{2}\right)\right)
$$

is the generator of a $D$-norm, called a Hüsler-Reiss D-norm. This norm depends only on the covariance matrix $\Sigma$.

There is a smallest $D$-norm and a largest one:

$$
\|\boldsymbol{x}\|_{\infty} \leq\|\boldsymbol{x}\|_{D} \leq\|\boldsymbol{x}\|_{1}, \quad \boldsymbol{x} \in \mathbb{R}^{d}
$$

see equation (1.4) in Falk (2019).

Note that the univariate distributions of the components $Z_{1}, \ldots, Z_{d}$ of $\boldsymbol{Z}$ influence the $D$-norm, which is generated by $\boldsymbol{Z}$. Suppose $Z_{1}, \ldots, Z_{d}$ are independent and identically distributed (iid) random variables. Then it makes a difference, whether they follow the standard exponential distribution or the uniform distribution on $(0,2)$.

Neither the generator of a $D$-norm is uniquely determined nor its distribution. Actually, if $\boldsymbol{Z}=\left(Z_{1}, \ldots, Z_{d}\right)$ is the generator of a $D$-norm, then $Z_{X}:=$ $\left(Z_{1} X, Z_{2} X, \ldots, Z_{d} X\right)$ generates the same $D$-norm if $X$ is a random variable with $X \geq 0$ and $E(X)=1$, which is also independent of $\boldsymbol{Z}$. But for any $D$-norm $\|\cdot\|_{D}$ on $\mathbb{R}^{d}$ and an arbitrary norm $\|\cdot\|$ on $\mathbb{R}^{d}$ there exists a generator $\boldsymbol{Z}$ of $\|\cdot\|_{D}$ with the additional property $\|\boldsymbol{Z}\|=$ const. The distribution of this generator is uniquely determined. This is the content of the following result, which is Theorem 1.7.1 in Falk (2019).

Theorem 2.3 (Normed Generators) Let $\|\cdot\|$ be an arbitrary norm on $\mathbb{R}^{d}$. For any D-norm $\|\cdot\|_{D}$ on $\mathbb{R}^{d}$, there exists a generator $\boldsymbol{Z}$ with the additional property $\|\boldsymbol{Z}\|=$ const. The distribution of this generator is uniquely determined.

The following consequence is Corollary 1.7.2 in Falk (2019).

Corollary 2.4 For any D-norm $\|\cdot 1\|_{D}$ on $\mathbb{R}^{d}$, there exist generators $\boldsymbol{Z}^{(1)}$, $\boldsymbol{Z}^{(2)}$ with the property $\left\|\boldsymbol{Z}^{(1)}\right\|_{1}=\sum_{i=1}^{d} Z_{i}^{(1)}=d$, and $\left\|\boldsymbol{Z}^{(2)}\right\|_{\infty}=\max _{1 \leq i \leq d} Z_{i}^{(2)}=$ const $=$ $\|(1, \ldots, 1)\|_{D}$.

The following characterization will be used in several proofs in this paper. It goes back to Takahashi (1987) and Takahashi (1988). For a proof see Corollary 1.3.5 in Falk (2019).

Corollary 2.5 Let $\|\cdot\|_{D}$ be an arbitrary D-norm on $\mathbb{R}^{d}$. We have the characterizations:

(i) $\|\cdot\|_{D}=\|\cdot\|_{1} \Longleftrightarrow \forall 1 \leq i<j \leq d:\left\|\boldsymbol{e}_{i}+\boldsymbol{e}_{j}\right\|_{D}=2=\left\|\boldsymbol{e}_{i}+\boldsymbol{e}_{j}\right\|_{1}$,

(ii) $\|\cdot\|_{D}=\|\cdot\|_{\infty} \Longleftrightarrow \exists i \in\{1, \ldots, d\} \forall j \neq i:\left\|\boldsymbol{e}_{i}+\boldsymbol{e}_{j}\right\|_{D}=1$. 


\section{When the number $E(h(Z))$ is generator invariant}

Let $\boldsymbol{Z}$ be the generator of a $D$-norm $\|\cdot\|_{D}$ on $\mathbb{R}^{d}$, and let $h:[0, \infty)^{d} \rightarrow[0, \infty)$ be a continuous function that is homogeneous of order one, i.e., $h(c \boldsymbol{y})=\operatorname{ch}(\boldsymbol{y}), c \geq 0$, $\boldsymbol{y} \in[0, \infty)^{d}$. In Theorem 3.5 we will establish the fact that, with such a function $h$, the number $E(h(\boldsymbol{Z}))$ does not depend on the particular generator $\boldsymbol{Z}$ of $\|\cdot\|_{D}$.

Take, for example, an arbitrary norm $\|\cdot\|$ on $\mathbb{R}^{d}$. With $h(\cdot)=\|\cdot\|$ we obtain that the number $E(\|\boldsymbol{Z}\|)$ does not depend on the particular generator $\boldsymbol{Z}$ of a $D$-norm $\|\cdot\|_{D}$. Equally, with $h(\boldsymbol{y}):=\min _{1 \leq i \leq d}\left(\left|x_{i}\right| y_{i}\right)$, Theorem 3.5 explains, why the function « $\boldsymbol{x} \gtrless_{D}=E\left(\min _{1 \leq i \leq d}\left|x_{i}\right| Z_{i}\right)=E(h(\boldsymbol{Z}))$, known as the dual D-norm function, is generator invariant.

Our proof of Theorem 3.5 uses several auxiliary results, which we establish first. They might be of interest of their own. We will frequently use the equation

$$
E(X)=\int_{0}^{\infty} P(X>u) d u
$$

valid for an arbitrary random variable $X \geq 0$; for a proof see, e.g., Lemma 1.2 in Falk (2019). By $1_{A}(\boldsymbol{x})$ we denote the indicator function of a set $A$, i.e., $1_{A}(\boldsymbol{x})=1$ if $x \in A$, and zero elsewhere. All operations on vectors are meant componentwise.

Lemma 3.1 Choose $\boldsymbol{y} \in[0, \infty)^{d}, \boldsymbol{y} \neq \mathbf{0} \in \mathbb{R}^{d}$, and $\boldsymbol{z}>\boldsymbol{y}$. Put $I^{*}:=\{j \in$ $\left.\{1, \ldots, d\}: y_{j}>0\right\}$. Then we have for $\boldsymbol{x} \in[0, \infty)^{d}$

$$
1_{[y, z)}(x)=\sum_{T \subset I^{*}}(-1)^{|T|-1} 1_{A_{T}}(\boldsymbol{x}),
$$

where $|T|$ denotes the cardinality of the set $T$, and

$$
A_{T}:=\left\{\boldsymbol{a} \in[0, \infty)^{d}: \max _{j \in T}\left(a_{j} / y_{j}\right) \geq 1\right\} \cup\left\{\boldsymbol{a} \in[0, \infty)^{d}: \max _{i \in T^{\complement}}\left(a_{i} / z_{i}\right) \geq 1\right\},
$$

with $T^{\complement}=\{1, \ldots, d\} \backslash T$.

Proof Choose $\boldsymbol{x} \in[0, \infty)^{d}$. Suppose first that $x_{i} \geq z_{i}$ for some $i \in\{1, \ldots, d\}$. Then we have $1_{[\boldsymbol{y}, z)}(\boldsymbol{x})=0$ and $1_{A_{T}}(\boldsymbol{x})=1$ for each $T \subset I^{*}$ and, thus,

$$
\sum_{T \subset I^{*}}(-1)^{|T|-1} 1_{A_{T}}(\boldsymbol{x})=\sum_{T \subset I^{*}}(-1)^{|T|-1}=0
$$

by the fact that

$$
1=\sum_{\emptyset \neq T \subset\{1, \ldots, n\}}(-1)^{|T|-1}
$$

for a proof see, e.g., equation (1.10) in Falk (2019). 
Suppose next that $x_{j}<y_{j}$ for some $j \in\{1, \ldots, d\}$. This implies $j \in I^{*}$; we have $1_{A_{T}}(\boldsymbol{x})=1_{A_{T \cup\{j\}}}(\boldsymbol{x})$ for each $T \subset I^{*}$ and, thus,

$$
\begin{aligned}
& \sum_{T \subset I^{*}}(-1)^{|T|-1} 1_{A_{T}}(\boldsymbol{x}) \\
= & \sum_{T \subset I^{*}, j \notin T}(-1)^{|T|-1} 1_{A_{T}}(\boldsymbol{x})+\sum_{T \subset I^{*}, j \in T}(-1)^{|T|-1} 1_{A_{T}}(\boldsymbol{x}) \\
= & \sum_{T \subset I^{*}, j \notin T}(-1)^{|T|-1} 1_{A_{T}}(\boldsymbol{x})+\sum_{T \subset I^{*}, j \notin T}(-1)^{|T|} 1_{A_{T \cup\{j\}}}(\boldsymbol{x}) \\
= & \sum_{T \subset I^{*}, j \notin T}(-1)^{|T|-1} 1_{A_{T}}(\boldsymbol{x})-\sum_{T \subset I^{*}, j \notin T}(-1)^{|T|-1} 1_{A_{T \cup\{j\}}}(\boldsymbol{x}) \\
= & \sum_{T \subset I^{*}, j \notin T}(-1)^{|T|-1}\left(1_{A_{T}}(\boldsymbol{x})-1_{A_{T \cup\{j\}}}(\boldsymbol{x})\right) \\
= & 0=1_{[\boldsymbol{y}, z)}(\boldsymbol{x}) .
\end{aligned}
$$

Suppose finally that $\boldsymbol{y} \leq \boldsymbol{x}<\boldsymbol{z}$. Then we have $\boldsymbol{x} \in A_{T}$ for each $T \neq \emptyset, \boldsymbol{x} \notin A_{\emptyset}$, and, thus, by Eq. 3.2

$$
\sum_{T \subset I^{*}}(-1)^{|T|-1} 1_{A_{T}}(\boldsymbol{x})=\sum_{\emptyset \neq T \subset I^{*}}(-1)^{|T|-1} 1_{A_{T}}(\boldsymbol{x})=1=1_{[\boldsymbol{y}, z)}(\boldsymbol{x}) .
$$

The measure $v$, introduced in the next result, is known as the exponent measure in MEVT; see, for example, Section 4 in Falk (2008). The definition of the exponent measure in equation (1.17) in Falk (2019) is, however, restricted to generators $\boldsymbol{Z}$ that realize in some unit sphere. Different to that, the measure $v$ in Eq. 3.3 supposes no restriction on $\boldsymbol{Z}$. This general definition is particulary required for the derivation of Theorem 3.5 below.

Proposition 3.2 Choose a D-norm $\|\cdot\|_{D}$ on $\mathbb{R}^{d}$ and an arbitrary generator $\boldsymbol{Z}$ of $\|\cdot\|_{D}$. Put $\boldsymbol{E}:=[0, \infty)^{d} \backslash\{\mathbf{0}\}$ and define the function $T:(0, \infty) \times \boldsymbol{E} \rightarrow \boldsymbol{E}$ by

$$
T(s, z):=\frac{1}{s} z, \quad s \in(0, \infty), z \in \boldsymbol{E} .
$$

Then

$$
\nu(\cdot):=\left(\lambda \times P_{\mathbf{Z}}\right)\left(T^{-1}(\cdot)\right)
$$

defines a measure on the Borel $\sigma$-field $\mathbb{B}_{\boldsymbol{E}}$ of $\boldsymbol{E}$ with

$$
v\left([\mathbf{0}, \boldsymbol{x}]^{\complement}\right)=\left\|\frac{\mathbf{1}}{\boldsymbol{x}}\right\|_{D}, \quad \boldsymbol{x}>\mathbf{0} \in \mathbb{R}^{d},
$$

where $\lambda$ denotes the Lebesgue measure on $(0, \infty), P_{Z}(\cdot)=P(Z \in \cdot)$ is the distribution of $\boldsymbol{Z}$, and " · $\times$." denotes the product measure. The measure $v$ on $\boldsymbol{E}$ is uniquely determined by Eq. 3.4.

The following consequence of Proposition 3.2 is obvious. 
Corollary 3.3 The measure $v$, as defined in Eq. 3.3, does not depend on the particular generator $\mathbf{Z}$ of $\|\cdot\|_{D}$.

Proof of Proposition 3.2 First we show that the measure $v$ satisfies Eq. 3.4. We have for $\boldsymbol{x}=\left(x_{1}, \ldots, x_{d}\right)>\mathbf{0} \in \mathbb{R}^{d}$

$$
\begin{aligned}
& v\left([\mathbf{0}, \boldsymbol{x}]^{\complement}\right) \\
= & \left(\lambda \times P_{\boldsymbol{Z}}\right)\left(\left\{(s, z): \frac{1}{s} z \in[\mathbf{0}, \boldsymbol{x}]^{\complement}\right\}\right) \\
= & \left(\lambda \times P_{\boldsymbol{Z}}\right)\left(\left\{(s, \boldsymbol{z}): \frac{1}{s} z_{i}>x_{i} \text { for some } i \in\{1, \ldots, d\}\right)\right. \\
= & \left(\lambda \times P_{\boldsymbol{Z}}\right)\left(\left\{(s, z): \max _{1 \leq i \leq d} \frac{z_{i}}{x_{i}}>s\right\}\right) \\
= & \int_{\boldsymbol{E}} \int_{0}^{\infty} 1\left(\left\{\max _{1 \leq i \leq d} \frac{z_{i}}{x_{i}}>s\right\}\right) d s P_{\boldsymbol{Z}}(d \boldsymbol{z}) \\
= & \int_{\boldsymbol{E}} \max _{1 \leq i \leq d} \frac{z_{i}}{x_{i}} P_{\boldsymbol{Z}}(d \boldsymbol{z}) \\
= & \int_{[0, \infty)^{d}} \max _{1 \leq i \leq d} \frac{z_{i}}{x_{i}} P_{\boldsymbol{Z}}(d z) \\
= & E\left(\max _{1 \leq i \leq d} \frac{Z_{i}}{x_{i}}\right)=\left\|\frac{\mathbf{1}}{\boldsymbol{x}}\right\|_{D} .
\end{aligned}
$$

Repeating the preceding arguments, one obtains

$$
v\left([\mathbf{0}, \boldsymbol{x})^{\complement}\right)=\left\|\frac{\mathbf{1}}{\boldsymbol{x}}\right\|_{D}, \quad \boldsymbol{x}>\mathbf{0} \in \mathbb{R}^{d},
$$

as well.

Let $\mu$ be another measure on $\mathbb{B}_{\boldsymbol{E}}$ which satisfies Eq. 3.4. We obtain for $\boldsymbol{y} \in$ $[0, \infty)^{d}, \boldsymbol{y} \neq \mathbf{0} \in \mathbb{R}^{d}$, and $z>\boldsymbol{y}$

$$
\begin{aligned}
v([\boldsymbol{y}, \boldsymbol{z})) & =\int_{\boldsymbol{E}} 1_{[\boldsymbol{y}, \boldsymbol{z})}(\boldsymbol{x}) v(d \boldsymbol{x}) \\
& =\int_{\boldsymbol{E}} \sum_{T \subset I^{*}}(-1)^{|T|-1} 1_{A_{T}}(\boldsymbol{x}) v(d \boldsymbol{x}) \quad \text { (use Lemma 3.1) } \\
& =\sum_{T \subset I^{*}}(-1)^{|T|-1} \int_{\boldsymbol{E}} 1_{A_{T}}(\boldsymbol{x}) v(d \boldsymbol{x}) \\
& =\sum_{T \subset I^{*}}(-1)^{|T|-1} v\left(A_{T}\right) \\
& =\sum_{T \subset I^{*}}(-1)^{|T|-1} v\left(\left[\mathbf{0}, \sum_{j \in T} y_{j} \boldsymbol{e}_{j}+\sum_{i \in T^{\complement}} z_{i} \boldsymbol{e}_{i}\right)^{\complement}\right)
\end{aligned}
$$




$$
\begin{aligned}
& =\sum_{T \subset I^{*}}(-1)^{|T|-1}\left\|\frac{1}{\sum_{j \in T} y_{j} \boldsymbol{e}_{j}+\sum_{i \in T^{\complement} z_{i} \boldsymbol{e}_{i}}}\right\|_{D} \\
& =\mu([\boldsymbol{y}, \boldsymbol{z}))
\end{aligned}
$$

by repeating the above arguments. The set of all intervals $[\boldsymbol{y}, \boldsymbol{z}), \boldsymbol{y} \geq \mathbf{0}, \boldsymbol{y} \neq \mathbf{0} \in \mathbb{R}^{d}$, and $\boldsymbol{z}>\boldsymbol{y}$, is intersection stable and generates the Borel $\sigma$-field $\mathbb{B}_{\boldsymbol{E}}$ of $\boldsymbol{E}$. Moreover, the measure $v$ is $\sigma$-finite with

$$
v\left(\left[\mathbf{0},\left(\frac{1}{n}, \ldots, \frac{1}{n}\right)\right)^{\complement}\right)=\left\|\frac{\mathbf{1}}{\left(\frac{1}{n}, \ldots, \frac{1}{n}\right)}\right\|_{D}=n\|\mathbf{1}\|_{D}
$$

for $n \in \mathbb{N}, \cup_{n \in \mathbb{N}}[\mathbf{0},(1 / n, \ldots, 1 / n))^{\complement}=\boldsymbol{E}$, and, thus, $v=\mu$ on $\mathbb{B}_{\boldsymbol{E}}$.

Lemma 3.4 The uniquely determined measure v in Proposition 3.2 satisfies

$$
v(c M)=\frac{1}{c} v(M), \quad c>0, M \in \mathbb{B}_{\boldsymbol{E}}
$$

Proof We have, with $c>0$ and $M \in \mathbb{B}_{\boldsymbol{E}}$,

$$
\begin{aligned}
v(c M) & =\left(\lambda \times P_{Z}\right)\left(\left\{(s, z): \frac{1}{s} z \in c M\right\}\right) \\
& =\left(\lambda \times P_{Z}\right)\left(\left\{(s, z): \frac{1}{s c} z \in M\right\}\right) \\
& =\int_{E} \int_{0}^{\infty} 1\left(\frac{1}{s c} z \in M\right) d s P_{Z}(d z) \quad \text { (use Fubini) } \\
& \left.=\frac{1}{c} \int_{E} \int_{0}^{\infty} 1\left(\frac{1}{s} z \in M\right) d s P_{Z}(d z) \quad \text { (by substituting } s \mapsto s / c\right) \\
& =\frac{1}{c} v(M) \quad \text { (use Fubini again). }
\end{aligned}
$$

The following theorem is the main result of this section. It shows that a wide class of functions $h:[0, \infty)^{d} \rightarrow[0, \infty)$ has the property that the number $E(h(\boldsymbol{Z}))$ is generator invariant. This result will also be a crucial tool in the proof of Theorem 4.4, which presents a new characterization of max-domain of attraction.

Theorem 3.5 Let $\boldsymbol{Z}^{(1)}, \boldsymbol{Z}^{(2)}$ be two generators of the D-norm $\|\cdot\|_{D}$ on $\mathbb{R}^{d}$. Then we have for each continuous function $h:[0, \infty)^{d} \rightarrow[0, \infty)$, which is homogeneous of order one, i.e., $h(c \boldsymbol{x})=\operatorname{ch}(\boldsymbol{x}), c \geq 0, \boldsymbol{x} \in[0, \infty)^{d}$,

$$
E\left(h\left(\boldsymbol{Z}^{(1)}\right)\right)=E\left(h\left(\boldsymbol{Z}^{(2)}\right)\right) \text {. }
$$


Proof With $T$ and $\lambda$ as in Proposition 3.2, the measures

$$
\begin{aligned}
& \nu(M):=\left(\lambda \times P_{Z}^{(1)}\right)\left(T^{-1}(M)\right) \\
& \mu(M):=\left(\lambda \times P_{Z}^{(2)}\right)\left(T^{-1}(M)\right)
\end{aligned}
$$

coincide on $\mathbb{B}_{\boldsymbol{E}}$ by Corollary 3.3. As a consequence we obtain

$$
\begin{aligned}
& v(\{\boldsymbol{x}: h(\boldsymbol{x}) \geq 1\}) \\
= & \left(\lambda \times P_{\boldsymbol{Z}^{(1)}}\right)\left(\left\{(s, z): h\left(\frac{1}{s} \boldsymbol{z}\right) \geq 1\right\}\right) \\
= & \left(\lambda \times P_{\boldsymbol{Z}^{(1)}}\right)(\{(s, z): h(z) \geq s\}) \\
= & \int_{\boldsymbol{E}} \int_{0}^{\infty} 1(h(z) \geq s) d s P_{\boldsymbol{Z}^{(1)}}(d \boldsymbol{z}) \quad \text { (use Fubini) } \\
= & \int_{\boldsymbol{E}} h(\boldsymbol{z}) P_{\boldsymbol{Z}^{(1)}}(d \boldsymbol{z}) \\
= & \left.\int_{[0, \infty)^{d}} h(\boldsymbol{z}) P_{\boldsymbol{Z}^{(1)}}(d \boldsymbol{z}) \quad \text { (because } h(\mathbf{0})=0\right) \\
= & E\left(h\left(\boldsymbol{Z}^{(1)}\right)\right) .
\end{aligned}
$$

Replacing $v$ by $\mu$ in the preceding list of equations yields the assertion, because $v=\mu$.

The preceding result explains, for example, why the dual $D$-norm function

$$
\text { ux }{\varkappa_{D}}_{D}:=E\left(\min _{1 \leq i \leq d}\left(\left|x_{i}\right| Z_{i}\right)\right), \quad \boldsymbol{x} \in \mathbb{R}^{d},
$$

does not depend on the particular generator $\boldsymbol{Z}=\left(Z_{1}, \ldots, Z_{d}\right)$ of $\|\cdot\|_{D}$. Just put, for $\boldsymbol{x}=\left(x_{1}, \ldots, x_{d}\right) \in \mathbb{R}^{d}$,

$$
h(\boldsymbol{y}):=\min _{1 \leq i \leq d}\left(\left|x_{i}\right| y_{i}\right), \quad \boldsymbol{y}=\left(y_{1}, \ldots, y_{d}\right) \in[0, \infty)^{d},
$$

in Theorem 3.5. For the significance of the dual $D$-norm function in terms of exceedance probabilities we refer to Falk (2019).

Another promising application of Theorem 3.5 is the co-extremality coefficient.

Definition 3.6 Take an arbitrary $D$-norm $\|\cdot\|_{D}$ on $\mathbb{R}^{d}$ with generator $\boldsymbol{Z}=$ $\left(Z_{1}, \ldots, Z_{d}\right)$. Then

$$
c_{i j}:=E\left(\sqrt{Z_{i} Z_{j}}\right)
$$

is the co-extremality coefficient, $1 \leq i, j \leq d$.

The co-extremality coefficient does not depend on the particular generator $\boldsymbol{Z}=$ $\left(Z_{1}, \ldots, Z_{d}\right)$ of $\|\cdot\|_{D}$; just put

$$
h(\boldsymbol{y}):=\sqrt{y_{i} y_{j}}
$$

in Theorem 3.5. 
The co-extremality coefficient turns out to be a measure of pairwise tail dependence, as revealed by the following result; see also Eq. 4.5 . Given a $D$-norm $\|\cdot\|_{D}$ on $\mathbb{R}^{d}$, with generator $\boldsymbol{Z}=\left(Z_{1}, \ldots, Z_{d}\right)$, we denote by

$$
\|(x, y)\|_{D_{i j}}:=\left\|x \boldsymbol{e}_{i}+y \boldsymbol{e}_{j}\right\|_{D}, \quad x, y \in \mathbb{R},
$$

the bivariate projection of $\|\cdot\|_{D}$, which is itself a $D$-norm, with generator

$$
Z^{(i, j)}:=\left(Z_{i}, Z_{j}\right), \quad 1 \leq i<j \leq d .
$$

Proposition 3.7 We have $c_{i j} \in[0,1], 1 \leq i, j \leq d$, with $c_{i i}=1$ and, for $i \neq j$,

(i) $\quad c_{i j}=0 \Longleftrightarrow\|\cdot\|_{D_{i j}}=\|\cdot\|_{1}$

(ii) $\quad c_{i j}=1 \Longleftrightarrow\|\cdot\|_{D_{i j}}=\|\cdot\|_{\infty}$.

Formulated in terms of random variables, the preceding result reads as follows. Suppose the random vector $\boldsymbol{\xi}=\left(\xi_{1}, \ldots, \xi_{d}\right)$ follows a simple max-stable $\mathrm{df} G(\boldsymbol{x})=$ $\exp \left(-\|\mathbf{1} / \boldsymbol{x}\|_{D}\right), \boldsymbol{x}>\mathbf{0} \in \mathbb{R}^{d}$, as in Theorem 4.1 below. Choose $1 \leq i<j \leq d$. Then $\xi_{i}, \xi_{j}$ are independent $\Longleftrightarrow c_{i j}=0$, and $\xi_{i}=\xi_{j}$ a.s. $\Longleftrightarrow c_{i j}=1$. The co-extremality coefficient is in this sense similar to the extremal dependence measure defined in Larsson and Resnick (2012). Another characterization of the coextremality coefficient in terms of a random vector $\boldsymbol{X}$, which is in the domain of attraction of $G$, is given in Eq. 4.5.

Proof We first establish part (i). Suppose that $c_{i j}=E\left(\sqrt{Z_{i} Z_{j}}\right)=0$. Then $Z_{i} Z_{j}=$ 0 a.s. and, thus, $\min \left(Z_{i}, Z_{j}\right)=0$ a.s. As a consequence,

$$
Z_{i}+Z_{j}=\max \left(Z_{i}, Z_{j}\right)+\min \left(Z_{i}, Z_{j}\right)=\max \left(Z_{i}, Z_{j}\right) \quad \text { a.s. }
$$

and thus, taking expectations, we obtain

$$
2=E\left(Z_{i}+Z_{j}\right)=E\left(\max \left(Z_{i}, Z_{j}\right)\right)=\|(1,1)\|_{D_{i j}} .
$$

Corollary 2.5 now implies $\|\cdot\|_{D_{i j}}=\|\cdot\|_{1}$.

If, on the other hand, $\|\cdot\|_{D_{i j}}=\|\cdot\|_{1}$, then we can choose by Theorem 3.5 for $\left(Z_{i}, Z_{j}\right)$ in $c_{i j}=E\left(\sqrt{Z_{i} Z_{j}}\right)$ the random permutation of $(2,0)$ with equal probability $1 / 2$. But then $Z_{i} Z_{j}=0$ and, thus, $c_{i j}=0$.

Next we establish part (ii). Suppose that $\|\cdot\|_{D_{i j}}=\|\cdot\|_{\infty}$. Then we can choose by Theorem 3.5 for $\left(Z_{i}, Z_{j}\right)$ in $c_{i j}=E\left(\sqrt{Z_{i} Z_{j}}\right)$ the constant random vector $(1,1)$ and obtain $c_{i j}=1$.

Now we prove the reverse direction. From Hölder's inequality we know that

$$
0 \leq c_{i j}=E\left(\sqrt{Z_{i}} \sqrt{Z_{j}}\right) \leq E\left(Z_{i}\right)^{1 / 2} E\left(Z_{j}\right)^{1 / 2}=1,
$$

with equality $c_{i j}=1$ iff $Z_{i}=Z_{j}$ a.s. But $\left(Z_{i}, Z_{j}\right)=\left(Z_{i}, Z_{i}\right)$ a.s. generates the bivariate $D$-norm $\|\cdot\|_{D_{i j}}=\|\cdot\|_{\infty}$.

The co-extremality coefficient behaves quite similar to the ordinary coefficient of correlation. But, while the ordinary coefficient of correlation is a measure of overall linear dependence in the data, the co-extremality coefficient addresses the upper tail 
of a bivariate distribution. Even more, the matrix of pairwise co-extremality coefficients turns out to be positive semidefinite, just like the ordinary correlation matrix. The following result might, therefore, enable the application of standard statistical techniques such as principal component analysis to extremal data. But this is future work. A principal component analysis for the extremal dependence measure defined in Larsson and Resnick (2012) was pursued by Cooley and Thibaud (2019).

Lemma 3.8 The co-extremality matrix

$$
C:=\left(c_{i j}\right)_{1 \leq i, j \leq d}
$$

is positive semidefinite.

Proof We have for $\boldsymbol{y}=\left(y_{1}, \ldots, y_{d}\right) \in \mathbb{R}^{d}$

$$
\begin{aligned}
\boldsymbol{y} C \boldsymbol{y}^{\top} & =\sum_{1 \leq i, j \leq d} y_{i} E\left(\sqrt{Z_{i} Z_{j}}\right) y_{j} \\
& =E\left(\sum_{1 \leq i, j \leq d} y_{i} \sqrt{Z_{i}} \sqrt{Z_{j}} y_{j}\right) \\
& =E\left(\left(\sum_{1 \leq i \leq d} y_{i} \sqrt{Z_{i}}\right)^{2}\right) \geq 0 .
\end{aligned}
$$

\section{Multivariate Extremes and D-Norms}

Next we link $D$-norms with MEVT. A df $G$ on $\mathbb{R}^{d}$ is called max-stable, if for every $n \in \mathbb{N}$ there exists vectors $\boldsymbol{a}_{n}>\mathbf{0}, \boldsymbol{b}_{n} \in \mathbb{R}^{d}$ such that

$$
G^{n}\left(\boldsymbol{a}_{n} \boldsymbol{x}+\boldsymbol{b}_{n}\right)=G(\boldsymbol{x}), \quad \boldsymbol{x} \in \mathbb{R}^{d} .
$$

Recall that all operations on vectors such as addition, multiplication etc. are always meant componentwise.

A df $G$ on $\mathbb{R}^{d}$ is a simple max-stable or simple extreme value df iff it is max-stable in the sense of Eq. 4.1, and if it has unit Fréchet margins:

$$
G\left(x \boldsymbol{e}_{i}\right)=G(0, \ldots, 0, x, 0, \ldots, 0)=\exp (-1 / x), \quad x>0,1 \leq i \leq d .
$$

In this case, the norming constants are $\boldsymbol{b}_{n}:=\mathbf{0} \in \mathbb{R}^{d}$ and $\boldsymbol{a}_{n}:=(n, \ldots, n) \in \mathbb{R}^{d}$. $n \in \mathbb{N}$.

The theory of $D$-norms allows a mathematically elegant characterization of an arbitrary simple max-stable df as formulated in the next result; for a proof see Theorem 2.3.3 in Falk (2019). It comes from results found in Balkema and Resnick (1977), de Haan and Resnick (1977), Pickands (1981), and Vatan (1985). By $1=$ $(1, \ldots, 1) \in \mathbb{R}^{d}$ we denote the vector in $\mathbb{R}^{d}$ with constant entry one. 
Theorem 4.1 A df G on $\mathbb{R}^{d}$ is a simple max-stable df iff there exists a D-norm $\|\cdot\|_{D}$ on $\mathbb{R}^{d}$ such that

$$
G(\boldsymbol{x})=\exp \left(-\left\|\frac{\mathbf{1}}{\boldsymbol{x}}\right\|_{D}\right), \quad \boldsymbol{x}>\mathbf{0} \in \mathbb{R}^{d} .
$$

A multivariate df $F$ on $\mathbb{R}^{d}$ is said to be in the max-domain of attraction of a simple EVD $G$, denoted by $F \in \mathcal{D}(G)$, if there are vectors $\boldsymbol{a}_{n}>\mathbf{0}, \boldsymbol{b}_{n} \in \mathbb{R}^{d}, n \in \mathbb{N}$, such that

$$
F^{n}\left(\boldsymbol{a}_{n} \boldsymbol{x}+\boldsymbol{b}_{n}\right) \longrightarrow_{n \rightarrow \infty} G(\boldsymbol{x}), \quad \boldsymbol{x} \in \mathbb{R}^{d} .
$$

This definition clearly applies to an arbitrary EVD $G$, but without loss of generality and in view of Theorem 4.1, we restrict our approach in what follows to the case of a simple EVD $G$.

Put $\boldsymbol{a}_{t}:=\boldsymbol{a}_{[t]}, \boldsymbol{b}_{t}:=\boldsymbol{b}_{[t]}, t \geq 1$, where $[t]:=\max \{n \in \mathbb{N}: n \leq t\}$ is the integer part of $t \geq 1$. Then the condition $F \in \mathcal{D}(G)$ in Eq. 4.2 can be formulated in a continuous way as follows. We have $F \in \mathcal{D}(G)$ iff there are vectors $\boldsymbol{a}_{t}>\mathbf{0} \in \mathbb{R}^{d}$, $\boldsymbol{b}_{t} \in \mathbb{R}^{d}, t \geq 1$, such that

$$
F^{t}\left(\boldsymbol{a}_{t} \boldsymbol{x}+\boldsymbol{b}_{t}\right) \rightarrow_{t \rightarrow \infty} G(\boldsymbol{x}), \quad \boldsymbol{x} \in \mathbb{R}^{d} .
$$

This equivalent condition will ease later proofs.

Recall that a copula on $\mathbb{R}^{d}$ is the df of a random vector $\boldsymbol{U}=\left(U_{1}, \ldots, U_{d}\right)$ with the property that each $U_{i}$ follows the uniform distribution on $(0,1)$. For an exhaustive account on copulas we refer to Nelsen (2006). Sklar's theorem (Sklar 1959, 1996) plays a major role for the characterization of $F \in \mathcal{D}(G)$.

Theorem 4.2 (Sklar) For an arbitrary $d f F$ on $\mathbb{R}^{d}$, with univariate margins $F_{1}, \ldots, F_{d}$, there exists a copula $C$ such that

$$
F(\boldsymbol{x})=C\left(F_{1}\left(x_{1}\right), \ldots, F_{d}\left(x_{d}\right)\right), \quad \boldsymbol{x}=\left(x_{1}, \ldots, x_{d}\right) \in \mathbb{R}^{d} .
$$

If $F$ is continuous, then $C$ is uniquely determined and given by

$$
C(\boldsymbol{u})=F\left(F_{1}^{-1}\left(u_{1}\right), \ldots, F_{d}^{-1}\left(u_{d}\right)\right), \quad \boldsymbol{u}=\left(u_{1}, \ldots, u_{d}\right) \in(0,1)^{d},
$$

where $F_{i}^{-1}(u)=\inf \left\{t \in \mathbb{R}: F_{i}(t) \geq u\right\}, u \in(0,1)$, is the generalized inverse of $F_{i}$. The copula of a random vector $\boldsymbol{Y}=\left(Y_{1}, \ldots, Y_{d}\right)$ is meant to be the copula of its df.

The next result goes back to Deheuvels (1984) and Galambos (1987). It is established in Proposition 3.1.10 in Falk (2019).

Proposition 4.3 A d-variate df $F$ satisfies $F \in \mathcal{D}(G)$ iff this is true for the univariate margins of $F$ together with the condition that the copula $C_{F}$ of $F$ satisfies the expansion

$$
C_{F}(\boldsymbol{u})=1-\|\mathbf{1}-\boldsymbol{u}\|_{D}+o(\|\mathbf{1}-\boldsymbol{u}\|)
$$

as $\boldsymbol{u} \rightarrow \mathbf{1}$, uniformly for $\boldsymbol{u} \in[0,1]^{d}$, where $\|\cdot\|_{D}$ is the D-norm on $\mathbb{R}^{d}$ that corresponds to $G$ in the sense of Theorem 4.1.

In the next result we present a new characterization of multivariate max-domain of attraction. 
Theorem 4.4 Let $\boldsymbol{X} \geq \mathbf{0} \in \mathbb{R}^{d}$ be a random vector with $d f F$. Then we have

$$
\begin{aligned}
F^{t}(t \boldsymbol{x}) & \rightarrow_{t \rightarrow \infty} \exp \left(-\left\|\frac{\mathbf{1}}{\boldsymbol{x}}\right\|_{D}\right), \quad \boldsymbol{x}>\mathbf{0} \in \mathbb{R}^{d}, \\
& \Longleftrightarrow t P(h(\boldsymbol{X})>t) \rightarrow_{t \rightarrow \infty} E(h(\boldsymbol{Z}))
\end{aligned}
$$

for each continuous function $h:[0, \infty)^{d} \rightarrow[0, \infty)$, which is homogeneous of order one, and $\mathbf{Z}$ is an arbitrary generator of the D-norm $\|\cdot\|_{D}$.

Note that by Theorem 3.5, the number $E(h(\boldsymbol{Z}))$ does not depend on the particular generator $\boldsymbol{Z}$ of $\|\cdot\|_{D}$.

Remark 4.5 The conclusion in the preceding result is obvious for $\boldsymbol{X}:=\boldsymbol{Z} / U$, where $\boldsymbol{Z}$ is an arbitrary generator of a $D$-norm $\|\cdot\|_{D}$, the random variable $U$ follows the uniform distribution on $(0,1)$, and $U$ and $\boldsymbol{Z}$ are independent. In this case, we obtain for each measurable function $h:[0, \infty)^{d} \rightarrow[0, \infty)$ which is homogeneous of order one,

$$
\begin{aligned}
t P\left(h\left(\frac{1}{U} \boldsymbol{Z}\right)>t\right) & =t P(h(\boldsymbol{Z})>t U) \\
& =t \int_{0}^{1} P(h(\boldsymbol{Z})>t u) d u \quad \text { (use Fubini) } \\
& =\int_{0}^{t} P(h(\boldsymbol{Z})>u) d u \\
& \rightarrow t \rightarrow \infty \int_{0}^{\infty} P(h(\boldsymbol{Z})>u) d u=E(h(\boldsymbol{Z}))
\end{aligned}
$$

by Eq. 3.1. Note again that by Theorem 3.5, the number $E(h(\boldsymbol{Z}))$ does not depend on the particular generator $\boldsymbol{Z}$ of $\|\cdot\|_{D}$.

If the generator $\boldsymbol{Z}$ is in addition bounded, then $\boldsymbol{X}=\boldsymbol{Z} / U$ follows a simple multivariate Generalized Pareto Distribution (GPD). In this case its df is $F_{\boldsymbol{X}}(\boldsymbol{x})=$ $1-\|\mathbf{1} / \boldsymbol{x}\|_{D}$ for large $\boldsymbol{x}>\mathbf{0} \in \mathbb{R}^{d}$. The definition of a multivariate GPD is not unique in the literature. But, if $\boldsymbol{Y}=\left(Y_{1}, \ldots, Y_{d}\right)$ is an arbitrary random vector whose copula is excursion stable and each component $Y_{i}$ follows in its upper tail a standard Pareto distribution, then we call its distribution a simple GPD. The df $F_{\boldsymbol{Y}}(\boldsymbol{x})$ of $\boldsymbol{Y}$ coincides for large $\boldsymbol{x}>\mathbf{0} \in \mathbb{R}^{d}$ in this case with that of $\boldsymbol{X}=\boldsymbol{Z} / U$; see Remark 3.1.3 in Falk (2019).

Proof of Theorem 4.4 The implication " $\Longleftarrow "$ is easily seen: Choose $\boldsymbol{x}=\left(x_{1}, \ldots\right.$, $\left.x_{d}\right)>\mathbf{0} \in \mathbb{R}^{d}$ and put $h(\boldsymbol{y}):=\max _{1 \leq i \leq d}\left(y_{i} / x_{i}\right)$. Then the assumption implies

$$
t P\left(\max _{1 \leq i \leq d} \frac{X_{i}}{x_{i}}>t\right) \rightarrow_{t \rightarrow \infty} E\left(\max _{1 \leq i \leq d} \frac{Z_{i}}{x_{i}}\right)=\left\|\frac{\mathbf{1}}{\boldsymbol{x}}\right\|_{D} .
$$

But

$$
\begin{gathered}
t P\left(\max _{1 \leq i \leq d} \frac{X_{i}}{x_{i}}>t\right)=t\left(1-P\left(\max _{1 \leq i \leq d} \frac{X_{i}}{x_{i}} \leq t\right)\right) \\
=t(1-F(t \boldsymbol{x}))
\end{gathered}
$$


and thus, we obtain from the fact that $\log (1+\varepsilon) / \varepsilon \rightarrow_{\varepsilon \rightarrow 0} 1$,

$$
\begin{aligned}
F^{t}(t \boldsymbol{x})= & \exp (t \log (F(t \boldsymbol{x}))) \\
= & \exp (t \log (1+(F(t \boldsymbol{x})-1))) \\
= & \exp \left(\frac{\log (1+(F(t \boldsymbol{x})-1))}{F(t \boldsymbol{x})-1} t(F(t \boldsymbol{x})-1)\right) \\
& \rightarrow_{t \rightarrow \infty} \exp \left(-\left\|\frac{\mathbf{1}}{\boldsymbol{x}}\right\|_{D}\right),
\end{aligned}
$$

which is the assertion.

Next we establish the implication " $\Longrightarrow$ ". Taking the logarithm on both sides of the assumption implies

$$
t \log (F(t \boldsymbol{x})) \rightarrow_{t \rightarrow \infty}-\left\|\frac{\mathbf{1}}{\boldsymbol{x}}\right\|_{D},
$$

or

$$
t \frac{\log (1+(F(t \boldsymbol{x})-1))}{F(t \boldsymbol{x})-1}(1-F(t \boldsymbol{x})) \rightarrow_{t \rightarrow \infty}\left\|\frac{\mathbf{1}}{\boldsymbol{x}}\right\|_{D},
$$

i.e.,

$$
t(1-F(t \boldsymbol{x})) \rightarrow_{t \rightarrow \infty}\left\|\frac{\mathbf{1}}{\boldsymbol{x}}\right\|_{D} .
$$

In Proposition 3.2 we defined a uniquely determined measure $v$ on the Borel $\sigma$ field $\mathbb{B}_{\boldsymbol{E}}$ of $\boldsymbol{E}=[0, \infty)^{d} \backslash\{\boldsymbol{0}\}$, with $v\left([\mathbf{0}, \boldsymbol{x}]^{\mathrm{C}}\right)=\|\mathbf{1} / \boldsymbol{x}\|_{D}, \boldsymbol{x}>\mathbf{0} \in \mathbb{R}^{d}$. Put, for $t>0$,

$$
v_{t}(M):=t P\left(\frac{1}{t} \boldsymbol{X} \in M\right), \quad M \in \mathbb{B}_{\boldsymbol{E}}
$$

Then $v_{t}, t>0$, defines a sequence of measures on $\mathbb{B}_{\boldsymbol{E}}$ with

$$
v_{t}\left([\mathbf{0}, \boldsymbol{x}]^{\complement}\right) \rightarrow_{t \rightarrow \infty}\left\|\frac{\mathbf{1}}{\boldsymbol{x}}\right\|_{D}=v\left([\mathbf{0}, \boldsymbol{x}]^{\complement}\right), \quad \boldsymbol{x}>\mathbf{0} \in \mathbb{R}^{d} .
$$

From Eqs. 3.5 and 3.6 we therefore obtain, for $\boldsymbol{y} \geq \mathbf{0} \in \mathbb{R}^{d}, \boldsymbol{y} \neq \mathbf{0}$, and $\boldsymbol{z}>\boldsymbol{y}$,

$$
v_{t}([\boldsymbol{y}, \boldsymbol{z})) \rightarrow_{t \rightarrow \infty} v([\boldsymbol{y}, \boldsymbol{z})) .
$$

But this implies that $v_{t}$ converges to $v$ vaguely as $t$ tends to infinity (Resnick 2008, Proposition 5.17), i.e., $v_{t}(M) \rightarrow_{t \rightarrow \infty} v(M)$ for each $M \in \mathbb{B}_{\boldsymbol{E}}$ with $v(\partial M)=0$, where $\partial M=\bar{M} \cap \overline{M^{\complement}}$ is the topological border of the set $M$, with $\bar{A}$ denoting the topological closure of a set $A \subset \boldsymbol{E}$.

Choose a continuous function $h:[0, \infty)^{d} \rightarrow[0, \infty)$, which is homogeneous of order one. Then we have, for $t>0$,

$$
t P(h(\boldsymbol{X})>t)=t P\left(h\left(\frac{1}{t} \boldsymbol{X}\right)>1\right)=v_{t}(M),
$$

with $M:=\{\boldsymbol{x} \in \boldsymbol{E}: h(\boldsymbol{x})>1\}$. If we can show that $\nu(\partial M)=0$, then we obtain $v_{t}(M) \rightarrow_{t \rightarrow \infty} v(M)$, or

$$
t P(h(X)>t) \rightarrow_{t \rightarrow \infty} v(M)
$$


First we claim that

$$
v(M)=E(h(\boldsymbol{Z})) .
$$

But this can easily be seen as follows:

$$
\begin{aligned}
v(M) & =\left(\lambda \times P_{\boldsymbol{Z}}\right)\left(T^{-1}(M)\right) \\
& =\left(\lambda \times P_{\boldsymbol{Z}}\right)\left(\left\{(s, z): \frac{1}{s} z \in M\right\}\right) \\
& =\left(\lambda \times P_{\boldsymbol{Z}}\right)\left(\left\{(s, z): h\left(\frac{1}{s} \boldsymbol{z}\right)>1\right\}\right) \\
& =\left(\lambda \times P_{\boldsymbol{Z}}\right)(\{(s, z): h(z)>s\}) \\
& =\int_{\boldsymbol{E}} \int_{0}^{\infty} 1(h(z)>s) d s P_{\boldsymbol{Z}}(d \boldsymbol{z}) \quad \text { (use Fubini) } \\
& =\int_{\boldsymbol{E}} h(\boldsymbol{z}) P_{\boldsymbol{Z}}(d \boldsymbol{z}) \\
& =\int_{[0, \infty)^{d}} h(\boldsymbol{z}) P_{\boldsymbol{Z}}(d \boldsymbol{z}) \quad(\text { as } h(\mathbf{0})=0) \\
& =E(h(\boldsymbol{Z})) .
\end{aligned}
$$

Therefore, all what is left to prove is that $\nu(\partial M)=0$. We have, by the continuity of the function $h$,

$$
\begin{aligned}
\partial M & =\bar{M} \cap \overline{M^{\complement}} \\
& =\{\boldsymbol{x} \in \boldsymbol{E}: h(\boldsymbol{x}) \geq 1\} \cap\{\boldsymbol{x} \in \boldsymbol{E}: h(\boldsymbol{x}) \leq 1\} \\
& =\{\boldsymbol{x} \in \boldsymbol{E}: h(\boldsymbol{x})=1\} .
\end{aligned}
$$

Finally, by repeating the arguments in the derivation of Eq. 4.4, we obtain

$$
v(\{\boldsymbol{x} \in \boldsymbol{E}: h(\boldsymbol{x}) \geq 1\})=v(\{\boldsymbol{x} \in \boldsymbol{E}: h(\boldsymbol{x})>1\})=E(h(\boldsymbol{Z})),
$$

and, thus,

$$
\begin{aligned}
v(\partial M) & =v(\{\boldsymbol{x} \in \boldsymbol{E}: h(\boldsymbol{x})=1\}) \\
& =v(\{\boldsymbol{x} \in \boldsymbol{E}: h(\boldsymbol{x}) \geq 1\} \backslash\{\boldsymbol{x} \in \boldsymbol{E}: h(\boldsymbol{x})>1\}) \\
& =v(\{\boldsymbol{x} \in \boldsymbol{E}: h(\boldsymbol{x}) \geq 1\})-v(\{\boldsymbol{x} \in \boldsymbol{E}: h(\boldsymbol{x})>1\})=0 .
\end{aligned}
$$

Recall that $E(h(\boldsymbol{Z}))<\infty$, as we can choose, by Theorem 2.3, a generator $\boldsymbol{Z}$ which is bounded. This completes the proof of Theorem 4.4 .

Theorem 4.4 implies, for example, the following characterization of the coextremality index:

$$
\begin{aligned}
c_{i j} & =E\left(\sqrt{Z_{i} Z_{j}}\right) \\
& =\lim _{t \rightarrow \infty} t P\left(X_{i} X_{j}>t^{2}\right)=\lim _{t \rightarrow \infty} \frac{P\left(X_{i} X_{j}>t^{2}\right)}{\sqrt{P\left(X_{i}>t\right) P\left(X_{j}>t\right)}}
\end{aligned}
$$

for $1 \leq i, j \leq d$, if the random vector $\boldsymbol{X}=\left(X_{1}, \ldots, X_{d}\right) \geq \mathbf{0} \in \mathbb{R}^{d}$ satisfies the first condition in Theorem 4.4. 
The fact that $t P\left(X_{i} X_{j}>t^{2}\right)$ has a limit if $t$ tends to infinity was already observed by (Jessen and Mikosch 2006, Section 4).

Another consequence of Theorem 4.4 is the following result, which was already established in Barbe et al. (2006). It is particularly interesting for risk assessment, as the event $\sum_{i=1}^{d} X_{i}>t$ with a large threshold $t$ may describe an unwanted exceedance above a high threshold. By Corollary 4.6, the probability of this event is approximately $d / t$ as $t$ increases, independent of the dependence structure among $X_{1}, \ldots, X_{d}$.

Corollary 4.6 Let $\boldsymbol{X}=\left(X_{1}, \ldots, X_{d}\right) \geq \mathbf{0} \in \mathbb{R}^{d}$ be a random vector that satisfies the first condition in Theorem 4.4. For continuous functions $h_{1}, \ldots, h_{n}:[0, \infty)^{d} \rightarrow$ $[0, \infty), n \in \mathbb{N}$, which are homogeneous of order one, we have

$$
\lim _{t \rightarrow \infty} t P\left(\sum_{i=1}^{n} h_{i}(\boldsymbol{X})>t\right)=\sum_{i=1}^{n} \lim _{t \rightarrow \infty} t P\left(h_{i}(\boldsymbol{X})>t\right)=\sum_{i=1}^{n} E\left(h_{i}(\boldsymbol{Z})\right) .
$$

As a special case we get

$$
\lim _{t \rightarrow \infty} t P\left(\sum_{i=1}^{d} X_{i}>t\right)=d,
$$

regardless of the tail dependence structure among $X_{1}, \ldots, X_{d}$.

Proof The function $h(\boldsymbol{x}):=\sum_{i=1}^{n} h_{i}(\boldsymbol{x})$ is continuous, non negative and homogeneous of order one. We can apply Theorem 4.4 and obtain

$$
\begin{aligned}
\lim _{t \rightarrow \infty} t P\left(\sum_{i=1}^{n} h_{i}(\boldsymbol{X})>t\right) & =E\left(\sum_{i=1}^{n} h_{i}(\boldsymbol{Z})\right) \\
& =\sum_{i=1}^{n} E\left(h_{i}(\boldsymbol{Z})\right) \\
& =\sum_{i=1}^{n} \lim _{t \rightarrow \infty} t P\left(h_{i}(\boldsymbol{X})>t\right) .
\end{aligned}
$$

By choosing $n=d$ and $h_{i}(\boldsymbol{x})=x_{i}, 1 \leq i \leq d, \boldsymbol{x}=\left(x_{1}, \ldots, x_{d}\right)$, we obtain the special case

$$
\lim _{t \rightarrow \infty} t P\left(\sum_{i=1}^{d} X_{i}>t\right)=\sum_{i=1}^{d} E\left(Z_{i}\right)=d
$$

Theorem 4.4 enables the following characterization, when a copula $C$ is in the max-domain of attraction of an EVD $G$. This time we consider a standard EVD $G(\boldsymbol{x})=\exp \left(-\|\boldsymbol{x}\|_{D}\right), \boldsymbol{x} \leq \mathbf{0} \in \mathbb{R}^{d}$, instead of a simple one. This is due to the fact that each univariate margin of $C$ is the uniform distribution on $(0,1)$, with $\mathrm{df}$ $H(x)=x, x \in[0,1]$. It satisfies $H\left(1-n^{-1} x\right) \rightarrow_{n \rightarrow \infty} \exp (x), x \leq 0$, and thus, if $C \in \mathcal{D}(G)$, then the EVD $G$ has necessarily standard exponential margins. In this 
case it can be represented as $G(\boldsymbol{x})=\exp \left(-\|\boldsymbol{x}\|_{D}\right), \boldsymbol{x} \leq \mathbf{0} \in \mathbb{R}^{d}$, with some $D$-norm $\|\cdot\|_{D}$, see Theorem 2.3.3 in Falk (2019).

Corollary 4.7 Suppose the random vector $\boldsymbol{U}=\left(U_{1}, \ldots, U_{d}\right)$ follows a copula $C$. We have

$$
\begin{aligned}
& C \in \mathcal{D}(G), \text { with } G(\boldsymbol{x})=\exp \left(-\|\boldsymbol{x}\|_{D}\right), \quad \boldsymbol{x} \leq \mathbf{0} \in \mathbb{R}^{d}, \\
& \Longleftrightarrow C^{t}\left(\mathbf{1}+\frac{1}{t} \boldsymbol{x}\right) \rightarrow_{t \rightarrow \infty} \exp \left(-\|\boldsymbol{x}\|_{D}\right), \quad \boldsymbol{x} \leq \mathbf{0} \in \mathbb{R}^{d}, \\
& \Longleftrightarrow t P\left(h\left(\frac{\mathbf{1}}{\mathbf{1}-\boldsymbol{U}}\right)>t\right) \rightarrow_{t \rightarrow \infty} E(h(\boldsymbol{Z})),
\end{aligned}
$$

for any continuous function $h:[0, \infty)^{d} \rightarrow[0, \infty)$, which is homogeneous of order one, with $\mathbf{Z}$ being an arbitrary generator of $\|\cdot\|_{D}$.

\section{Modeling Multivariate Exceedances}

The following result can be used for stochastic modeling of multivariate exceedances above high thresholds and the estimation of its probability. By $\rightarrow_{D}$ we denote ordinary convergence in distribution of random vectors or, equivalently, ordinary weak convergence of their corresponding distributions. For a thorough presentation of multivariate Peaks-over-Threshold modelling we refer to Rootzén et al. (2018) and the exhaustive literature cited therein.

Theorem 5.1 Let the random vector $\boldsymbol{X} \geq \mathbf{0} \in \mathbb{R}^{d}$ have df $F$ with

$$
F^{t}(t \boldsymbol{x}) \rightarrow_{t \rightarrow \infty} \exp \left(-\left\|\frac{\mathbf{1}}{\boldsymbol{x}}\right\|_{D}\right), \quad \boldsymbol{x}>\mathbf{0} \in \mathbb{R}^{d} .
$$

For an arbitrary norm $\|\cdot\|$ on $\mathbb{R}^{d}$, there exists by Theorem 2.3 a generator $\boldsymbol{Z}$ of $\|\cdot\|_{D}$ with the additional property $\|\mathbf{Z}\|=$ const. Its distribution $P_{\mathbf{Z}}$ is uniquely determined, and we have

$$
P\left(\text { const } \frac{\boldsymbol{X}}{\|\boldsymbol{X}\|} \in \cdot\|\boldsymbol{X}\|>t\right) \rightarrow_{D} P_{\boldsymbol{Z}}(\cdot)
$$

as $t \rightarrow \infty$.

The preceding result is in particular applicable with the choice $\|\cdot\|=\|\cdot\|_{1}$, in which case the typically unknown number const $=\|\boldsymbol{Z}\|_{1}$ equals $d$, just see the proof of Corollary 2.4.

Corollary 5.2 Under the conditions of Theorem 5.1 we obtain, with $\|\cdot\|=\|\cdot\|_{1}$,

$$
P\left(d \frac{\boldsymbol{X}}{\|\boldsymbol{X}\|_{1}} \in \cdot\|\boldsymbol{X}\|_{1}>t\right) \rightarrow_{D} P_{\boldsymbol{Z}}(\cdot)
$$

as $t \rightarrow \infty$, where $\boldsymbol{Z}$ is a generator of $\|\cdot\|_{D}$ with $\|\boldsymbol{Z}\|_{1}=d$. 
If we put in particular $\boldsymbol{X}:=\mathbf{1} /(\mathbf{1}-\boldsymbol{U})$, where $\boldsymbol{U}$ follows a copula $C \in \mathcal{D}(G)$, $G(\boldsymbol{x})=\exp \left(-\|\boldsymbol{x}\|_{D}\right), \boldsymbol{x} \leq \mathbf{0} \in \mathbb{R}^{d}$, then we obtain the above conclusion.

If a multivariate exceedance above a high threshold is defined as a realization of the random vector $\boldsymbol{X}=\left(X_{1}, \ldots, X_{d}\right) \geq \mathbf{0} \in \mathbb{R}^{d}$, with $\|\boldsymbol{X}\|_{1}=X_{1}+\cdots+X_{d} \geq t$ and a large value $t$, then Corollary 5.2 provides its asymptotic distribution. Having independent copies $\boldsymbol{X}^{(1)}, \ldots, \boldsymbol{X}^{(n)}$ of $\boldsymbol{X}$, the limit distribution $\boldsymbol{P}_{\boldsymbol{Z}}$ in Corollary 5.2 can be estimated in a straightforward manner by the ordinary empirical measure corresponding to $\boldsymbol{X}^{(1)}, \ldots, \boldsymbol{X}^{(n)}$ as follows. Put, for $\boldsymbol{x} \geq \mathbf{0} \in \mathbb{R}^{d}$ and $t>0$,

$$
F_{n, t}(\boldsymbol{x}):=\frac{1}{M_{n, t}} \sum_{i=1}^{n} 1\left(d \frac{\boldsymbol{X}^{(i)}}{\left\|\boldsymbol{X}^{(i)}\right\|_{1}} \leq \boldsymbol{x}\right) 1\left(\left\|\boldsymbol{X}^{(i)}\right\|_{1}>t\right),
$$

with

$$
M_{n, t}:=\sum_{i=1}^{n} 1\left(\left\|\boldsymbol{X}^{(i)}\right\|_{1}>t\right) .
$$

According to Lemma 1.4.1 in Reiss (1993), $F_{n, t}$ is the ordinary empirical df of $m$ independent and identically distributed random vectors $\boldsymbol{Y}^{(1)}, \ldots, \boldsymbol{Y}^{(m)}$ with $\mathrm{df}$

$$
P\left(\boldsymbol{Y}^{(i)} \leq \boldsymbol{x}\right)=P\left(d \frac{\boldsymbol{X}}{\|\boldsymbol{X}\|_{1}} \leq \boldsymbol{x} \mid\|\boldsymbol{X}\|_{1}>t\right), \quad 1 \leq i \leq m,
$$

conditional on $M_{n, t}=m$, where $M_{n, t}$ is also independent of $\boldsymbol{Y}^{(1)}, \ldots, \boldsymbol{Y}^{(m)}$. This representation allows the estimation of the distribution $P_{Z}$ as $n$ and $t$ both tend to infinity in a straightforward manner. But details are outside the scope of this paper and require future work.

In the proof of Theorem 5.1 we make use of the following auxiliary topological result.

Lemma 5.3 Choose an arbitrary norm $\|\cdot\|$ on $\mathbb{R}^{d}$ and let $A$ be a Borel subset of the sphere $S:=\left\{\boldsymbol{x} \in[0, \infty)^{d}:\|\boldsymbol{x}\|=1\right\}$. Put

$$
\begin{aligned}
M_{A} & :=(1, \infty) \cdot A:=\{s \boldsymbol{a}: s>1, \boldsymbol{a} \in A\} \\
& =\left\{\boldsymbol{x} \in[0, \infty)^{d}: \frac{\boldsymbol{x}}{\|\boldsymbol{x}\|} \in A,\|\boldsymbol{x}\|>1\right\} .
\end{aligned}
$$

Then, with $M_{A}$ seen as a subset of $[0, \infty)^{d}$,

$$
\partial M_{A} \subset S \cup(1, \infty) \cdot(\bar{A} \cap \overline{S \backslash A}) .
$$

Proof Let $\boldsymbol{x} \in \partial M_{A}$. Then $\|\boldsymbol{x}\| \geq 1$. If $\|\boldsymbol{x}\|=1$, then $\boldsymbol{x} \in S$ and we are done. So assume $\|\boldsymbol{x}\|>1$. Because $\boldsymbol{x} \in \partial M_{A}$, there exist sequences $\boldsymbol{x}_{n} \in M_{A}, n \in \mathbb{N}$, and $\boldsymbol{y}_{n} \notin M_{A}, n \in \mathbb{N}$, with

$$
\boldsymbol{x}=\lim _{n \in \mathbb{N}} \boldsymbol{x}_{n}=\lim _{n \in \mathbb{N}} \boldsymbol{y}_{n}
$$

which implies

$$
\lim _{n \in \mathbb{N}}\left\|\boldsymbol{x}_{n}\right\|=\lim _{n \in \mathbb{N}}\left\|\boldsymbol{y}_{n}\right\|=\|\boldsymbol{x}\|>1
$$


Without loss of generality we can assume $\left\|\boldsymbol{x}_{n}\right\|>1,\left\|\boldsymbol{y}_{n}\right\|>1, n \in \mathbb{N}$, and, thus, $\boldsymbol{x}_{n} /\left\|\boldsymbol{x}_{n}\right\| \in A, \boldsymbol{y}_{n} /\left\|\boldsymbol{y}_{n}\right\| \in S \backslash A, n \in \mathbb{N}$. The representation

$$
\frac{\boldsymbol{x}}{\|\boldsymbol{x}\|}=\lim _{n \in \mathbb{N}} \frac{\boldsymbol{x}_{n}}{\left\|\boldsymbol{x}_{n}\right\|}=\lim _{n \in \mathbb{N}} \frac{\boldsymbol{y}_{n}}{\left\|\boldsymbol{y}_{n}\right\|}
$$

then implies that $\boldsymbol{x} /\|\boldsymbol{x}\| \in \bar{A} \cap \overline{S \backslash A}$ and thus, $\boldsymbol{x} \in(1, \infty) \cdot(\bar{A} \cap \overline{S \backslash A})$, because $\|x\|>1$.

Proof of Theorem 5.1 We have to establish the convergence

$$
P\left(\operatorname{const} \frac{\boldsymbol{X}}{\|\boldsymbol{X}\|} \in M \mid\|X\|>t\right) \rightarrow_{t \rightarrow \infty} P_{\boldsymbol{Z}}(M)
$$

for each Borel subset $M \subset[0, \infty)^{d}$ with $P_{\boldsymbol{Z}}(\partial M)=P(\boldsymbol{Z} \in \partial M)=0$.

In the proof of Theorem 4.4 we established the convergence $v_{t}(M) \rightarrow_{t \rightarrow \infty} v(M)$ for each Borel set $M \in \mathbb{B}_{\boldsymbol{E}}, \boldsymbol{E}=[0, \infty)^{d} \backslash\{\boldsymbol{0}\}$, with $\nu(\partial M)=0$, where

$$
v(\cdot)=\left(\lambda \times P_{\mathbf{Z}}\right)\left(T^{-1}(\cdot)\right)
$$

as defined in Theorem 3.5, and

$$
v_{t}(\cdot)=t P\left(\frac{1}{t} \boldsymbol{X} \in \cdot\right) .
$$

Put

$$
S_{\text {const }}:=\left\{x \in[0, \infty)^{d}:\|x\|=\text { const }\right\}
$$

note that the generator $\boldsymbol{Z}$ realizes in $S_{\text {const }}$. Let $M \subset[0, \infty)^{d}$ be a Borel subset with $P(\boldsymbol{Z} \in \partial M)=0$. Put $A:=M \cap S_{\text {const }}$ and

$$
M_{A}:=(1, \infty) \cdot A=\{s \boldsymbol{a}: s>1, \boldsymbol{a} \in A\} .
$$

Check that $M_{A}$ is a Borel subset of $[0, \infty)^{d}$. Note that $\mathbf{0} \notin M_{A}$, because $\mathbf{0} \notin S_{\text {const }}$, and hence, $M_{A} \in \mathbb{B}_{\boldsymbol{E}}$.

From Lemma 5.3, applied to the norm $\|\cdot\| /$ const, we obtain

$$
\partial M_{A} \subset S_{\text {const }} \cup(1, \infty) \cdot\left(\bar{A} \cap \overline{S_{\text {const }} \backslash A}\right),
$$

which implies

$$
v\left(\partial M_{A}\right) \leq v\left(S_{\text {const }}\right)+v\left((1, \infty) \cdot\left(\bar{A} \cap \overline{S_{\text {const }} \backslash A}\right)\right) .
$$

In what follows we prove that the upper bound in this inequality vanishes.

We have

$$
v\left(S_{\text {const }}\right)=v\left(\left\{\boldsymbol{x} \geq \mathbf{0}: \frac{\boldsymbol{x}}{\text { const }} \geq 1\right\}\right)-v\left(\left\{\boldsymbol{x} \geq \mathbf{0}: \frac{\boldsymbol{x}}{\text { const }}>1\right\}\right)=0
$$


by the arguments in Eq. 4.4, and

$$
\begin{aligned}
& v\left((1, \infty) \cdot\left(\bar{A} \cap \overline{S_{\text {const }} \backslash A}\right)\right) \\
= & \left(\lambda \times P_{\boldsymbol{Z}}\right)\left(\left\{(s, z): \frac{1}{s} z \in(1, \infty) \cdot\left(\bar{A} \cap \overline{S_{\text {const }} \backslash A}\right)\right\}\right) \\
= & \int_{0}^{\infty} \int_{S_{\text {const }}} 1\left(z \in(s, \infty) \cdot\left(\bar{A} \cap \overline{S_{\text {const }} \backslash A}\right)\right) P_{\boldsymbol{Z}}(d z) d s \quad\left(\boldsymbol{Z} \text { is in } S_{\text {const }}\right) \\
= & \int_{0}^{1} \int_{\bar{A} \cap \overline{S_{\text {const }} \backslash A}} 1 P_{\boldsymbol{Z}}(d z) d s \quad\left(\boldsymbol{Z} \text { is in } S_{\text {const }}\right) \\
= & P\left(\boldsymbol{Z} \in\left(\bar{A} \cap \overline{S_{\text {const }} \backslash A}\right)\right) \\
\leq & \left.P(\boldsymbol{Z} \in \partial M) \quad \text { (because } \bar{A} \cap \overline{S_{\text {const }} \backslash A} \subset \partial M\right) \\
= & 0 \quad
\end{aligned}
$$

by assumption.

As a consequence of the preceding considerations we have $v(\partial M)=0$ and, thus,

$$
v_{t}(M) \rightarrow_{t \rightarrow \infty} v(M)
$$

From Theorem 4.4 we obtain

$$
\begin{aligned}
& \lim _{t \rightarrow \infty} P\left(\operatorname{const} \frac{\boldsymbol{X}}{\|\boldsymbol{X}\|} \in M \mid\|\boldsymbol{X}\|>t\right) \\
& =\lim _{t \rightarrow \infty} P\left(\operatorname{const} \frac{\boldsymbol{X}}{\|\boldsymbol{X}\|} \in M \mid\|\boldsymbol{X}\|>\text { const } t\right) \\
& =\lim _{t \rightarrow \infty} P\left(\text { const } \frac{\boldsymbol{X}}{\|\boldsymbol{X}\|} \in A \mid\|\boldsymbol{X}\|>\text { const } t\right) \quad \text { (recall that } A=M \cap S_{\text {const }} \text { ) } \\
& =\lim _{t \rightarrow \infty} \frac{P\left(\|X\|>\text { const } t, \text { const } \frac{X}{\|X\|} \in A\right)}{P(\|X\|>\text { const } t)} \\
& =\lim _{t \rightarrow \infty} \frac{P\left(X \in t M_{A}\right)}{P(\|X\|>\text { const } t)} \quad \text { (check equality of the sets) } \\
& =\frac{v\left(M_{A}\right)}{E(\|\boldsymbol{Z}\|) / \text { const }} \\
& =v\left(M_{A}\right) \quad(\text { recall that }\|\boldsymbol{Z}\|=\text { const }) \\
& =P(Z \in A) \quad \text { (repeat the arguments leading to Eq. 5.1). }
\end{aligned}
$$

This completes the proof of Theorem 5.1.

Remark 5.4 Theorem 5.1 is implied by Theorem 4.4, but not vice versa. Put $\boldsymbol{X}=$ $(X, X)$ with $P(X>t)=1 / \sqrt{t}, t \geq 1$, and set $Z=(1,1)$, which generates the bivariate $D$-norm $\|\cdot\|_{\infty}$.

With an arbitrary norm $\|\cdot\|$ on $\mathbb{R}^{2}$ we have $\|\boldsymbol{Z}\|=\|(1,1)\|=$ : const, and thus,

$$
\text { const } \frac{\boldsymbol{X}}{\|\boldsymbol{X}\|}=\mathrm{const} \frac{X(1,1)}{\|X(1,1)\|}=(1,1) \text {. }
$$


For all sets $M \in \mathbb{B}_{\boldsymbol{E}}$, and all $t>0$ this implies

$$
\begin{aligned}
& P\left(\text { const } \frac{\boldsymbol{X}}{\|\boldsymbol{X}\|} \in M \mid\|\boldsymbol{X}\|>t\right)= \begin{cases}1, & \text { if }(1,1) \in M, \\
0 & \text { elsewhere, }\end{cases} \\
& =P(Z \in M) \text {. }
\end{aligned}
$$

We thus have in particular that the limit result in Theorem 5.1 is valid, but, with $h\left(x_{1}, x_{2}\right)=x_{1}$,

$$
\lim _{t \rightarrow \infty} t P(X>t)=\lim _{t \rightarrow \infty} \sqrt{t} \neq E\left(Z_{1}\right)=1
$$

\section{On Generators Whose Components Sum up to d}

In this section we will see that Theorem 5.1 provides a way, how to simulate a generator $\boldsymbol{Z}^{(1)}=\left(Z_{1}^{(1)}, \ldots, Z_{d}^{(1)}\right)$ of an arbitrary $D$-norm $\|\cdot\|_{D}$ on $\mathbb{R}^{d}$, with the additional property $\left\|\boldsymbol{Z}^{(1)}\right\|_{1}=\sum_{i=1}^{d} Z_{i}^{(1)}=d$, and that it delivers the distribution of $\boldsymbol{Z}^{(1)}$, which is unique by Theorem 2.3 .

Take a $D$-norm $\|\cdot\|_{D}$ on $\mathbb{R}^{d}$, choose some generator $\boldsymbol{Z}=\left(Z_{1}, \ldots, Z_{d}\right)$ of it, and let $U$ be a random variable, which follows the uniform distribution on $(0,1)$, and which is independent of $\boldsymbol{Z}$. Put

$$
\boldsymbol{X}:=\frac{1}{U} \boldsymbol{Z}
$$

Then the random vector $\boldsymbol{X} \geq \mathbf{0} \in \mathbb{R}^{d}$ satisfies

$$
P(\boldsymbol{X} \leq t \boldsymbol{x})^{t} \rightarrow_{t \rightarrow \infty} \exp \left(-\left\|\frac{\mathbf{1}}{\boldsymbol{x}}\right\|_{D}\right), \quad \boldsymbol{x}>\mathbf{0} \in \mathbb{R}^{d},
$$

see Remark 4.5, and thus we obtain from Corollary 5.2

$$
P\left(d \frac{\boldsymbol{X}}{\|\boldsymbol{X}\|_{1}} \in \cdot\|\boldsymbol{X}\|_{1}>t\right) \rightarrow_{D} P\left(\boldsymbol{Z}^{(1)} \in \cdot\right)
$$

as $t \rightarrow \infty$. But $\boldsymbol{X} /\|\boldsymbol{X}\|_{1}=\boldsymbol{Z} /\|\boldsymbol{Z}\|_{1}$ and, thus,

$$
P\left(d \frac{\boldsymbol{X}}{\|\boldsymbol{X}\|_{1}} \in \cdot\|\boldsymbol{X}\|_{1}>t\right)=P\left(d \frac{\boldsymbol{Z}}{\|\boldsymbol{Z}\|_{1}} \in \cdot\|\boldsymbol{Z}\|_{1}>t U\right) .
$$

Choosing a large threshold $t>0$, this equation enables the simulation of $\boldsymbol{Z}^{(1)}$ in an obvious way. We will see below, in Proposition 6.2, that even the exact distribution of $\boldsymbol{Z}^{(1)}$ can be derived from this equation as well.

Example 6.1 Take, for example, the Dirichlet $D$-norm $\|\cdot\|_{D(\alpha)}$, with parameter $\alpha>$ 0 . It has the generator

$$
\boldsymbol{Z}=\left(Z_{1}, \ldots, Z_{d}\right)=\frac{1}{\alpha}\left(V_{1}, \ldots, V_{d}\right),
$$

where $V_{1}, \ldots, V_{d}$ are independent and identically gamma distributed random variables with density $\gamma_{\alpha}(x)=x^{\alpha-1} \exp (-x) / \Gamma(\alpha), x>0$. It is well known that 
the random variables $\left(V_{i} / \sum_{j=1}^{d} V_{j}\right)_{i=1}^{d}$ and the sum $\sum_{j=1}^{d} V_{j}$ are independent, see, e.g., the proof of Theorem 2.1 in $\mathrm{Ng}$ et al. (2011), and thus, by the independence of $\left(V_{1}, \ldots, V_{d}\right)$ and $U$,

$$
\begin{aligned}
& P\left(d \frac{\boldsymbol{Z}}{\|\boldsymbol{Z}\|_{1}} \in \cdot\|\boldsymbol{Z}\|_{1}>t U\right) \\
= & P\left(d\left(\frac{V_{1}}{\sum_{j=1}^{d} V_{j}}, \ldots, \frac{V_{d}}{\sum_{j=1}^{d} V_{j}}\right) \in \cdot \mid \sum_{j=1}^{d} V_{j}>\alpha t U\right) \\
= & P\left(d\left(\frac{V_{1}}{\sum_{j=1}^{d} V_{j}}, \ldots, \frac{V_{d}}{\sum_{j=1}^{d} V_{j}}\right) \in \cdot\right) \\
= & P\left(\boldsymbol{Z}^{(1)} \in \cdot\right)
\end{aligned}
$$

with $\boldsymbol{Z}^{(1)}:=d\left(V_{i} / \sum_{j=1}^{d} V_{j}\right)_{i=1}^{d}$.

Suppose, on the other hand, that the generator $\boldsymbol{Z}$ in the definition $\boldsymbol{X}=\boldsymbol{Z} / U$ already satisfies $\|\boldsymbol{Z}\|_{1}=d$. Then,

$$
\begin{aligned}
P\left(d \frac{\boldsymbol{X}}{\|\boldsymbol{X}\|_{1}} \in \cdot\|\boldsymbol{X}\|_{1}>t\right) & =P\left(d \frac{\boldsymbol{Z}}{\|\boldsymbol{Z}\|_{1}} \in \cdot\|\boldsymbol{Z}\|_{1}>t U\right) \\
& =P\left(\boldsymbol{Z} \in \cdot \mid \frac{d}{t}>U\right) \\
& =P(\boldsymbol{Z} \in \cdot), \quad t>0 .
\end{aligned}
$$

Theorem 5.1 also provides the distribution of $\boldsymbol{Z}^{(1)}$, given in the following result. This is actually one version of the angular measure, see (Falk 2019, Lemma 1.7.5), and Proposition 6.3 below.

Proposition 6.2 Take an arbitrary D-norm $\|\cdot\|_{D}$ on $\mathbb{R}^{d}$ and choose some generator $\boldsymbol{Z}$ of it. Then the distribution of a generator $\boldsymbol{Z}^{(1)}$ of $\|\cdot\|_{D}$, with $\left\|\boldsymbol{Z}^{(1)}\right\|_{1}=d$, equals

$$
P\left(\boldsymbol{Z}^{(1)} \in \cdot\right)=\frac{1}{d} E\left(1\left(d \frac{\boldsymbol{Z}}{\|\boldsymbol{Z}\|_{1}} \in \cdot\right)\|\boldsymbol{Z}\|_{1}\right) .
$$

Proof It is easy to see that

$$
Q(\cdot):=\frac{1}{d} E\left(1\left(d \frac{\boldsymbol{Z}}{\|\boldsymbol{Z}\|_{1}} \in \cdot\right)\|\boldsymbol{Z}\|_{1}\right)
$$

defines a probability measure on $[0, \infty)^{d}$ (use the monotone convergence theorem), with $Q\left(\left\{\boldsymbol{x} \geq \mathbf{0} \in \mathbb{R}^{d}:\|\boldsymbol{x}\|_{1}=d\right\}\right)=1$. To prove that the distribution of $\boldsymbol{Z}^{(1)}$ equals $Q(\cdot)$, we only have to show that their corresponding df coincide on $[0, \infty)^{d}$. 
From Theorem 5.1 we know that, with $\boldsymbol{X}:=\boldsymbol{Z} / U$, where $U$ is uniformly on $(0,1)$ distributed and independent of $\boldsymbol{Z}$,

$$
\begin{aligned}
& P\left(\boldsymbol{Z}^{(1)} \leq \boldsymbol{x}\right) \\
= & \lim _{t \rightarrow \infty} P\left(d \frac{\boldsymbol{X}}{\|\boldsymbol{X}\|_{1}} \leq \boldsymbol{x} \mid\|\boldsymbol{X}\|_{1}>t\right) \\
= & \lim _{t \rightarrow \infty} P\left(d \frac{\boldsymbol{Z}}{\|\boldsymbol{Z}\|_{1}} \leq \boldsymbol{x} \mid U<\frac{\|\boldsymbol{Z}\|_{1}}{t}\right) \\
= & \lim _{t \rightarrow \infty} \frac{P\left(d \frac{\boldsymbol{Z}}{\|\boldsymbol{Z}\|_{1}} \leq \boldsymbol{x}, U<\frac{\|\boldsymbol{Z}\|_{1}}{t}\right)}{P\left(U<\frac{\|\boldsymbol{Z}\|_{1}}{t}\right)},
\end{aligned}
$$

for each point $\boldsymbol{x} \in[0, \infty)^{d}$, at which the df of $\boldsymbol{Z}^{(1)}$ is continuous.

First we analyze the denominator in the above equation. We have

$$
\begin{aligned}
& P\left(U<\frac{\|\boldsymbol{Z}\|_{1}}{t}\right) \\
= & \int_{\left\{z:\|z\|_{1}>t\right\}} P\left(U<\frac{\|z\|_{1}}{t}\right) P_{\boldsymbol{Z}}(d z) \\
& +\int_{\left\{z:\|z\|_{1} \leq t\right\}} P\left(U<\frac{\|z\|_{1}}{t}\right) P_{Z}(d z) \\
= & \int_{\left\{z:\|z\|_{1}>t\right\}} 1 P_{\boldsymbol{Z}}(d z) \quad+\int_{\left\{z:\|z\|_{1} \leq t\right\}} \frac{\|z\|_{1}}{t} P_{Z}(d z) \\
= & P\left(\|\boldsymbol{Z}\|_{1}>t\right)+\frac{1}{t} \int_{\left\{z:\|z\|_{1} \leq t\right\}}\|z\|_{1}(Z(d z)
\end{aligned}
$$

The fact that $E\left(\|\boldsymbol{Z}\|_{1}\right)=d<\infty$ implies

$$
t P\left(\|\boldsymbol{Z}\|_{1}>t\right) \rightarrow_{t \rightarrow \infty} 0
$$

(use Lemma 3.1), and

$$
\begin{aligned}
& \int_{\left\{z:\|z\|_{1} \leq t\right\}}\|z\|_{1} P_{\boldsymbol{Z}}(d z) \\
& \rightarrow_{t \rightarrow \infty} \int_{[0, \infty)^{d}}\|z\|_{1} P_{\boldsymbol{Z}}(d z)=E\left(\|\boldsymbol{Z}\|_{1}\right)=d .
\end{aligned}
$$

As a consequence we obtain

$$
P\left(\|\boldsymbol{Z}\|_{1}>t\right)+\frac{1}{t} \int_{\left\{z:\|z\|_{1} \leq t\right\}}\|z\|_{1} P_{Z}(d z)=\frac{1}{t}(d+o(1)) .
$$


Next we investigate the numerator:

$$
\begin{aligned}
& P\left(d \frac{\boldsymbol{Z}}{\|\boldsymbol{Z}\|_{1}} \leq \boldsymbol{x}, U<\frac{\|\boldsymbol{Z}\|_{1}}{t}\right) \\
= & \iint_{0}^{1} 1\left(d \frac{\boldsymbol{z}}{\|z\|_{1}} \leq \boldsymbol{x}, u<\frac{\|z\|_{1}}{t}\right) d u P_{\boldsymbol{Z}}(d z) \\
= & \iint_{0}^{1} 1\left(d \frac{\boldsymbol{z}}{\|z\|_{1}} \leq \boldsymbol{x}\right) \times 1\left(u<\frac{\|z\|_{1}}{t}\right) d u P_{\boldsymbol{Z}}(d z) \\
= & \int 1\left(d \frac{\boldsymbol{z}}{\|z\|_{1}} \leq \boldsymbol{x}\right)\left[\int_{0}^{1} 1\left(u<\frac{\|z\|_{1}}{t}\right) d u\right] P_{\boldsymbol{Z}}(d z) \\
= & \int\left\{z:\|z\|_{1} \leq t\right\} \\
& +\int_{\left\{z:\|z\|_{1}>t\right\}} 1\left(d \frac{\boldsymbol{z}}{\|z\|_{1}} \leq \boldsymbol{x}\right) \frac{\|z\|_{1}}{t} P_{\boldsymbol{Z}}(d z) \\
= & \frac{1}{t}\left[E\left(1\left(d \frac{\boldsymbol{Z}}{\|\boldsymbol{Z}\|_{1}} \leq \boldsymbol{x}\right)\|\boldsymbol{Z}\|_{1}\right)+o(1)\right]+o\left(\frac{1}{t}\right) \\
= & \frac{1}{t}\left[E\left(1\left(d \frac{\boldsymbol{Z}}{\|\boldsymbol{Z}\|_{1}} \leq \boldsymbol{x}\right)\|\boldsymbol{Z}\|_{1}\right)+o(1)\right] .
\end{aligned}
$$

As a consequence we obtain

$$
\begin{aligned}
F(\boldsymbol{x}) & :=P\left(\boldsymbol{Z}^{(1)} \leq \boldsymbol{x}\right) \\
& =\lim _{t \rightarrow \infty} P\left(d \frac{\boldsymbol{Z}}{\|\boldsymbol{Z}\|_{1}} \leq \boldsymbol{x} \mid U<\frac{\|\boldsymbol{Z}\|_{1}}{t}\right) \\
& =\frac{1}{d} E\left(1\left(d \frac{\boldsymbol{Z}}{\|\boldsymbol{Z}\|_{1}} \leq \boldsymbol{x}\right)\|\boldsymbol{Z}\|_{1}\right)=: \tilde{F}(\boldsymbol{x})
\end{aligned}
$$

for each $\boldsymbol{x} \geq \mathbf{0} \in \mathbb{R}^{d}$, at which the df $F$ is continuous. But, being df, the functions $F$ and $\tilde{F}$ are both upper continuous and, therefore, $F(\boldsymbol{x})=\tilde{F}(\boldsymbol{x})$ for each $\boldsymbol{x} \in[0, \infty)^{d}$, and thus, $Q$ is the distribution of $\boldsymbol{Z}^{(1)}$.

The arguments in the proof of Proposition 6.2 can be repeated to establish in general the distribution of a generator $\tilde{\boldsymbol{Z}}$ of a $D$-norm $\|\cdot\|_{D}$, with the particular property $\|\tilde{\boldsymbol{Z}}\|=$ const, given an arbitrary norm $\|\cdot\|$ on $\mathbb{R}^{d}$.

Proposition 6.3 Take an arbitrary D-norm $\|\cdot\|_{D}$ on $\mathbb{R}^{d}$ and an arbitrary generator $\boldsymbol{Z}$ of $\|\cdot\|_{D}$. For each norm $\|\cdot\|$ on $\mathbb{R}^{d}$ there exists by Theorem 2.3 a generator $\tilde{\boldsymbol{Z}}$ of $\|\cdot\|_{D}$, with the additional property $\|\tilde{\boldsymbol{Z}}\|=$ const. Its uniquely determined distribution is given by

$$
P(\tilde{\boldsymbol{Z}} \in \cdot)=\frac{1}{\text { const }} E\left(1\left(\operatorname{const} \frac{\boldsymbol{Z}}{\|\boldsymbol{Z}\|} \in \cdot\right)\|\boldsymbol{Z}\|\right) .
$$


Note that the distribution of $\tilde{\boldsymbol{Z}}$ coincides with the angular measure in general, see (Falk 2019, Lemma 1.7.5).

If the arbitrary generator $\boldsymbol{Z}$ in Proposition 6.3 is bounded, then the distribution of $\tilde{Z}$ can be specified as follows. Note that the preceding result implies

$$
\text { const }=E(\|\boldsymbol{Z}\|) \text {. }
$$

Proposition 6.4 Suppose in addition to the assumptions in Proposition 6.3 that the arbitrary generator $\boldsymbol{Z}$ of $\|\cdot\|_{D}$ is bounded, i.e., $\|\boldsymbol{Z}\| \leq m$ for some number $m>0$. Then we have

$$
P(\tilde{\boldsymbol{Z}} \in \cdot)=P\left(\operatorname{const} \frac{\boldsymbol{Z}}{\|\boldsymbol{Z}\|} \in \cdot \mid\|\boldsymbol{Z}\|>U\right),
$$

where $U$ is a random variable that is independent of $\boldsymbol{Z}$ and which is uniformly distributed on the interval $(0, m)$.

Proof According to Proposition 6.3, we have to establish the equation

$$
P\left(\operatorname{const} \frac{\boldsymbol{Z}}{\|\boldsymbol{Z}\|} \in \cdot \mid\|\boldsymbol{Z}\|>U\right)=\frac{1}{\text { const }} E\left(1\left(\operatorname{const} \frac{\boldsymbol{Z}}{\|\boldsymbol{Z}\|} \in\right)\|\boldsymbol{Z}\|\right) .
$$

First we have, using Fubini's theorem,

$$
\begin{aligned}
P(\|\boldsymbol{Z}\|>U) & =\iint_{0}^{m} 1(u<\|z\|) \frac{1}{m} d u P_{\boldsymbol{Z}}(d z) \\
& =\iint_{0}^{\|z\|} \frac{1}{m} d u P_{\boldsymbol{Z}}(d z) \\
& =\frac{1}{m} \int\|z\| P_{\boldsymbol{Z}}(d z) \\
& =\frac{1}{m} E(\|\boldsymbol{Z}\|)=\frac{\text { const }}{m} .
\end{aligned}
$$

Using very similar arguments we obtain

$$
\begin{aligned}
& P\left(\text { const } \frac{\boldsymbol{Z}}{\|\boldsymbol{Z}\|} \in \cdot\|\boldsymbol{Z}\|>U\right) \\
= & \iint_{0}^{m} 1\left(\operatorname{const} \frac{z}{\|z\|} \in \cdot\right) 1(u<\|z\|) \frac{1}{m} d u P_{\boldsymbol{Z}}(d z) \\
= & \int 1\left(\operatorname{const} \frac{z}{\|z\|} \in \cdot\right) \int_{0}^{\|z\|} \frac{1}{m} d u P_{\boldsymbol{Z}}(d z) \\
= & \frac{1}{m} \int 1\left(\operatorname{const} \frac{z}{\|z\|} \in \cdot\right)\|z\| P_{\boldsymbol{Z}}(d z) \\
= & \frac{1}{m} E\left(1\left(\operatorname{const} \frac{\boldsymbol{Z}}{\|\boldsymbol{Z}\|} \in \cdot\right)\|\boldsymbol{Z}\|\right) .
\end{aligned}
$$

The above two equations now imply the assertion. 
The preceding result has the following consequence. If we know a bounded generator $\boldsymbol{Z}$ of a $D$-norm $\|\cdot\|_{D}$ and we are able to simulate it, then we can also simulate any normed generator $\tilde{\boldsymbol{Z}}$ of $\|\cdot\|_{D}$ with the following algorithm; recall that $\|\boldsymbol{Z}\| \leq m$.

1. Sample a realization $\boldsymbol{z}$ of $\boldsymbol{Z}$.

2. Sample a realization $u$ from the uniform distribution on $(0, m)$.

(a) If $\|z\| \leq u$ : Go back to step 1 .

(b) Else: Stop and return $\tilde{z}=$ const $\frac{z}{\|z\|}$.

This accept-reject algorithm runs with a random number of steps. As seen in the proof of Proposition 6.4, the probability of stopping is const/ $m$ for each iteration, so on average it takes $\mathrm{m} / \mathrm{const}$ iterations to stop. Note that in the particular case $\|\cdot\|=\|\cdot\|_{1}$, we have const $=d$ by the fact that $E\left(Z_{i}\right)=1$ for each component $Z_{i}$ of $\boldsymbol{Z}=\left(Z_{1}, \ldots, Z_{d}\right)$.

\section{On the Structure of Exchangeable D-Norms}

In the paper (Mai and Scherer 2020), the authors show that the set of $d$-variate symmetric stable tail dependence functions, uniquely associated with exchangeable $d$-dimensional extreme value copulas, is a simplex and they determine its extremal boundary.

It turns out that Mai and Scherer (2020) is actually a contribution to the theory of $D$-norms, as shown in what follows. To the best of our knowledge, we introduce the concept of an exchangeable $D$-norm for the first time, and we prove new results for this subfamily of $D$-norms.

Definition 7.1 A norm $\|\cdot\|$ on $\mathbb{R}^{d}$ is called exchangeable, if

$$
\left\|\left(x_{1}, \ldots, x_{d}\right)\right\|=\left\|\left(x_{\sigma(1)}, \ldots, x_{\sigma(d)}\right)\right\|, \quad \boldsymbol{x}=\left(x_{1}, \ldots, x_{d}\right) \in \mathbb{R}^{d},
$$

for each permutation $(\sigma(1), \ldots, \sigma(d))$ of $(1, \ldots, d)$.

Obvious examples are the sup-norm $\|\boldsymbol{x}\|_{\infty}=\max _{1 \leq i \leq d}\left|x_{i}\right|$ and each logistic norm $\|\boldsymbol{x}\|_{p}=\left(\sum_{i=1}^{d}\left|x_{i}\right|^{p}\right)^{1 / p}, p \geq 1$. The norm $\|\boldsymbol{x}\|=\sum_{i=1}^{d} \frac{1}{i}\left|x_{i}\right|$ is not exchangeable.

Recall that a random vector $\boldsymbol{X}=\left(X_{1}, \ldots, X_{d}\right)$ is said to have exchangeable components, if

$$
\left(X_{1}, \ldots, X_{d}\right)={ }_{D}\left(X_{\sigma(1)}, \ldots, X_{\sigma(d)}\right)
$$

for each permutation $(\sigma(1), \ldots, \sigma(d))$ of $(1, \ldots, d)$. By $=_{D}$ we denote equality in distribution. In this case we say that the random vector $\boldsymbol{X}$ is exchangeable. An obvious example is the case, where the components $X_{1}, \ldots, X_{d}$ are iid.

Recall that for each $D$-norm $\|\cdot\|_{D}$ on $\mathbb{R}^{d}$ and each norm $\|\cdot\|$ on $\mathbb{R}^{d}$ there exists by Theorem 2.3 a generator $\boldsymbol{Z}$ of $\|\cdot\|_{D}$, with the additional property $\|\boldsymbol{Z}\|=$ const. The distribution of this generator $\boldsymbol{Z}$ is uniquely determined. The name exchangeable D-norm is due to the following characterization. 
Theorem 7.1 Let $\|\cdot\|$ be an arbitrary exchangeable norm on $\mathbb{R}^{d}$. A D-norm $\|\cdot\|_{D}$ on $\mathbb{R}^{d}$ is exchangeable iff the generator $\boldsymbol{Z}$ of $\|\cdot\|_{D}$ with $\|\boldsymbol{Z}\|=$ const is exchangeable.

The reverse implication can be weakened considerable: If there exists a generator $\boldsymbol{Z}$ of $\|\cdot\|_{D}$, which is exchangeable, then $\|\cdot\|_{D}$ is exchangeable.

Proof The reverse implication is easily seen. Suppose there is a generator $\boldsymbol{Z}=\left(Z_{1}, \ldots, Z_{d}\right)$ of $\|\cdot\|_{D}$, which is exchangeable. Then we obtain, for $\boldsymbol{x}=$ $\left(x_{1}, \ldots, x_{d}\right) \in \mathbb{R}^{d}$,

$$
\begin{aligned}
\|\boldsymbol{x}\|_{D} & =E\left(\max _{1 \leq i \leq d}\left(\left|x_{i}\right| Z_{i}\right)\right)=E\left(\max _{1 \leq i \leq d}\left(\left|x_{i}\right| Z_{\sigma^{-1}(i)}\right)\right) \\
& =E\left(\max _{1 \leq i \leq d}\left(\left|x_{\sigma(i)}\right| Z_{i}\right)\right)=\left\|\left(x_{\sigma(1)}, \ldots, x_{\sigma(d)}\right)\right\|_{D},
\end{aligned}
$$

where $\sigma^{-1}$ is the inverse permutation of $\sigma$, i.e., $\sigma\left(\sigma^{-1}(i)\right)=i, 1 \leq i \leq d$.

Next we establish the implication " $\Longrightarrow$ ". Suppose that $\|\cdot\|_{D}$ is an exchangeable $D$-norm on $\mathbb{R}^{d}$. Choose an arbitrary exchangeable norm $\|\cdot\|$ on $\mathbb{R}^{d}$. There exists by Theorem 2.3 a generator $\tilde{Z}=\left(\tilde{Z}_{1}, \ldots, \tilde{Z}_{d}\right)$ of $\|\cdot\|_{D}$, with the additional property $\|\tilde{\boldsymbol{Z}}\|=$ const. Its distribution is uniquely determined. The fact that $\|\cdot\|_{D}$ is exchangeable implies, for $\boldsymbol{x} \in \mathbb{R}^{d}$, and an arbitrary permutation $\sigma=(\sigma(1), \ldots, \sigma(d))$ of $(1, \ldots, d)$,

$$
\begin{aligned}
\|\boldsymbol{x}\|_{D} & =\left\|\left(x_{1}, \ldots, x_{d}\right)\right\|_{D}=\left\|\left(x_{\sigma(1)}, \ldots, x_{\sigma(d)}\right)\right\|_{D} \\
& =E\left(\max _{1 \leq i \leq d}\left(\left|x_{\sigma(i)}\right| \tilde{Z}_{i}\right)\right)=E\left(\max _{1 \leq i \leq d}\left(\left|x_{i}\right| \tilde{Z}_{\sigma^{-1}(i)}\right)\right) .
\end{aligned}
$$

That means that

$$
\tilde{\boldsymbol{Z}}_{\sigma^{-1}}:=\left(\tilde{Z}_{\sigma^{-1}(1)}, \ldots, \tilde{Z}_{\sigma^{-1}(d)}\right)
$$

is a generator of $\|\cdot\|_{D}$ as well, with the property $\left\|\tilde{\boldsymbol{Z}}_{\sigma^{-1}}\right\|=\|\tilde{\boldsymbol{Z}}\|=$ const, due to the exchangeability of $\|\cdot\|$. But the distribution of this generator $\tilde{Z}$ is uniquely determined, and, thus,

$$
\tilde{Z}_{\sigma^{-1}}={ }_{D} \tilde{\boldsymbol{Z}}
$$

As $\sigma$ is an arbitrary permutation of $(1, \ldots, d)$, we have established exchangeability of $\tilde{\boldsymbol{Z}}$.

Remark 7.2 One might guess that each generator $\boldsymbol{Z}$ of an exchangeable $D$-norm is exchangeable. But this is not true. Take a random variable $U$, which follows the uniform distribution on $(0,1)$, and choose arbitrary numbers $a_{0}:=0<a_{1}<\cdots<$ $a_{d-1}<a_{d}:=1$. Then $\boldsymbol{Z}:=\left(Z_{1}, \ldots, Z_{d}\right)$ with

$$
Z_{i}:=\frac{1}{a_{i}-a_{i-1}} 1\left(U \in\left(a_{i-1}, a_{i}\right]\right), \quad 1 \leq i \leq d,
$$

is a generator of the $D$-norm $\|\cdot\|_{1}$, which is easily seen. But the generator $\boldsymbol{Z}$ is exchangeable only if $a_{i}-a_{i-1}=1 / d, 1 \leq i \leq d$. 
In what follows we will characterize extremal exchangeable $D$-norms. Let $\sigma^{*}=$ $\left(\sigma^{*}(1), \ldots, \sigma^{*}(d)\right)$ be a random permutation of $(1, \ldots, d)$, with equal probability $1 / d$ ! for each possible outcome, and denote by $\mathcal{P}_{d}$ the set of all permutations of $(1, \ldots, d)$.

Choose $\boldsymbol{t}=\left(t_{1}, \ldots, t_{d}\right) \in S_{d}:=\left\{\boldsymbol{s} \in[0,1]^{d}:\|\boldsymbol{s}\|_{1}=d\right\}$ and put, for $\boldsymbol{x} \in \mathbb{R}^{d}$,

$$
\begin{aligned}
\|\boldsymbol{x}\|_{D_{t}} & :=\frac{1}{d !} \sum_{\sigma \in \mathcal{P}_{d}} \max _{1 \leq i \leq d}\left(\left|x_{\sigma(i)}\right| t_{i}\right)=\frac{1}{d !} \sum_{\sigma \in \mathcal{P}_{d}} \max _{1 \leq i \leq d}\left(\left|x_{i}\right| t_{\sigma(i)}\right) \\
& =E\left(\max _{1 \leq i \leq d}\left(\left|x_{i}\right| t_{\sigma^{*}(i)}\right)\right) .
\end{aligned}
$$

Then $\|\cdot\|_{D_{t}}$ is an exchangeable $D$-norm on $\mathbb{R}^{d}$, with generator

$$
Z_{\boldsymbol{t}}:=\left(Z_{\boldsymbol{t}, 1}, \ldots, Z_{\boldsymbol{t}, d}\right):=\left(t_{\sigma^{*}(1)}, \ldots, t_{\sigma^{*}(d)}\right) .
$$

We obviously have $Z_{t, i}=t_{\sigma^{*}(i)} \geq 0$, and

$$
\begin{aligned}
E\left(Z_{t, i}\right) & =E\left(t_{\sigma^{*}(i)}\right)=\sum_{j=1}^{d} t_{j} P\left(\sigma^{*}(i)=j\right) \\
& =\sum_{j=1}^{d} t_{j} \frac{\text { number of permutations } \sigma \text { in } \mathcal{P}_{d} \text { with } \sigma(i)=j}{\text { total number of permutations in } \mathcal{P}_{d}} \\
& =\sum_{j=1}^{d} t_{j} \frac{(d-1) !}{d !}=\frac{1}{d} \sum_{j=1}^{d} t_{j}=\frac{d}{d}=1 .
\end{aligned}
$$

The $D$-norm $\|\cdot\|_{D_{t}}$ is obviously exchangeable.

The set of $D$-norms is convex, see (Falk 2019, Proposition 1.4.1), and, therefore, the set of exchangeable $D$-norms is convex as well. It turns out that the norms $\|\cdot\|_{D_{t}}$, $t \in S_{d}$, are extremal points of it. This is the content of our next result.

Lemma 7.1 Each D-norm $\|\cdot\|_{D_{t}}$, with $\boldsymbol{t} \in S_{d}$, is an extremal D-norm within the set of exchangeable D-norms on $\mathbb{R}^{d}$, i.e., if, for some $\lambda \in(0,1)$,

$$
\|\cdot\|_{D_{t}}=\lambda\|\cdot\|_{D^{(1)}}+(1-\lambda)\|\cdot\|_{D^{(2)}},
$$

where $\|\cdot\|_{D^{(1)}},\|\cdot\|_{D^{(2)}}$ are exchangeable D-norms on $\mathbb{R}^{d}$, then

$$
\|\cdot\|_{D^{(1)}}=\|\cdot\|_{D^{(2)}}=\|\cdot\|_{D_{t}} .
$$

Proof Let $\boldsymbol{Z}^{(i)}$ be a generator of $\|\cdot\|_{D^{(i)}}$, with the additional property $\left\|\boldsymbol{Z}^{(i)}\right\|_{1}=d$, $i=1,2$. Let $\xi \in\{1,2\}$ be a random variable with $P(\xi=1)=1-P(\xi=$ 2 ), and which is independent of $\boldsymbol{Z}^{(1)}$ and $\boldsymbol{Z}^{(1)}$. Then the random vector $\boldsymbol{Z}^{(\xi)}=$ $\left(Z_{1}^{(\xi)}, \ldots, Z_{d}^{(\xi)}\right)$ satisfies, for $\boldsymbol{x}=\left(x_{1}, \ldots, x_{d}\right) \in \mathbb{R}^{d}$,

$$
E\left(\max _{1 \leq i \leq d}\left(\left|x_{i}\right| Z_{i}^{(\xi)}\right)\right)=\lambda\|\boldsymbol{x}\|_{D^{(1)}}+(1-\lambda)\|\boldsymbol{x}\|_{D^{(2)}}=\|\boldsymbol{x}\|_{D_{t}},
$$


see the proof of Proposition 1.4.1 in Falk (2019). The generator $\boldsymbol{Z}^{(\xi)}$ realizes in $S_{d}$ as well, and thus, as the distribution of this generator is by Theorem 2.3 unique, its distribution coincides with that of $\boldsymbol{Z}_{\boldsymbol{t}}$. We, consequently, have

$$
P\left(\boldsymbol{Z}^{(\xi)}=\left(t_{\sigma(1)}, \ldots, t_{\sigma(d)}\right)\right)=P\left(\boldsymbol{Z}_{\boldsymbol{t}}=\left(t_{\sigma(1)}, \ldots, t_{\sigma(d)}\right)\right)=\frac{1}{d !}
$$

for each permutation $\sigma=(\sigma(1), \ldots, \sigma(d)) \in \mathcal{P}_{d}$. But this implies, with $\boldsymbol{t}_{\sigma}:=$ $\left(t_{\sigma(1)}, \ldots, t_{\sigma(d)}\right)$,

$$
\begin{aligned}
& \lambda \sum_{\sigma \in \mathcal{P}_{d}} P\left(\boldsymbol{Z}^{(1)}=\boldsymbol{t}_{\sigma}\right)+(1-\lambda) \sum_{\sigma \in \mathcal{P}_{d}} P\left(\boldsymbol{Z}^{(2)}=\boldsymbol{t}_{\sigma}\right) \\
= & \sum_{\sigma \in \mathcal{P}_{d}}\left(\lambda P\left(\boldsymbol{Z}^{(1)}=\boldsymbol{t}_{\sigma}\right)+(1-\lambda) P\left(\boldsymbol{Z}^{(2)}=\boldsymbol{t}_{\sigma}\right)\right) \\
= & \sum_{\sigma \in \mathcal{P}_{d}} P\left(\boldsymbol{Z}^{(\xi)}=\boldsymbol{t}_{\sigma}\right)=\sum_{\sigma \in \mathcal{P}_{d}} \frac{1}{d !}=1,
\end{aligned}
$$

and, thus,

$$
\sum_{\sigma \in \mathcal{P}_{d}} P\left(Z^{(1)}=\boldsymbol{t}_{\sigma}\right)=1=\sum_{\sigma \in \mathcal{P}_{d}} P\left(\boldsymbol{Z}^{(2)}=\boldsymbol{t}_{\sigma}\right) .
$$

Because $\boldsymbol{Z}^{(1)}$ and $\boldsymbol{Z}^{(2)}$ are exchangeable by Theorem 7.2, this implies

$$
P\left(Z^{(1)}=\boldsymbol{t}_{\sigma}\right)=\frac{1}{d !}=P\left(\boldsymbol{Z}^{(2)}=\boldsymbol{t}_{\sigma}\right)
$$

for each $\sigma \in \mathcal{P}_{d}$, i.e., $\boldsymbol{Z}^{(1)}={ }_{D} \boldsymbol{Z}^{(2)}={ }_{D} \boldsymbol{Z}_{\boldsymbol{t}}$, which is the assertion.

Our next result shows that the set of exchangeable $D$-norms on $\mathbb{R}^{d}$ is a simplex. It parallels Lemma 2.4 in Mai and Scherer (2020).

Lemma 7.2 Let $\|\cdot\|_{D}$ be an arbitrary exchangeable D-norm on $\mathbb{R}^{d}$. Then we have, for $\boldsymbol{x} \in \mathbb{R}^{d}$, the representation

$$
\|\boldsymbol{x}\|_{D}=\int_{S_{d}}\|\boldsymbol{x}\|_{D_{t}} P_{\tilde{\mathbf{Z}}}(d \boldsymbol{t})=E\left(\|\boldsymbol{x}\|_{D_{\tilde{\mathbf{Z}}}}\right),
$$

where $\tilde{\boldsymbol{Z}}$ is a generator of $\|\cdot\|_{D}$ with $\|\tilde{\boldsymbol{Z}}\|_{1}=d$, i.e., $\tilde{\boldsymbol{Z}}$ realizes in $S_{d}$. As the distribution of $\tilde{\boldsymbol{Z}}$ is by Theorem 2.3 uniquely determined, the above representation of $\|\cdot\|_{D}$ in terms of $\|\cdot\|_{D_{t}}, \boldsymbol{t} \in S_{d}$, and the distribution of $\tilde{\boldsymbol{Z}}$ is uniquely determined as well. 
Proof The assertion is easily seen. Conditioning on $\tilde{Z}=\boldsymbol{t}$, one obtains from the exchangeability of $\|\cdot\|_{D}$

$$
\begin{aligned}
& \|\boldsymbol{x}\|_{D}=E\left(\max _{1 \leq i \leq d}\left(\left|x_{i}\right| \tilde{Z}_{i}\right)\right) \\
& =\frac{1}{d !} \sum_{\sigma \in \mathcal{P}_{d}} E\left(\max _{1 \leq i \leq d}\left(\left|x_{\sigma(i)}\right| \tilde{Z}_{i}\right)\right) \\
& =\frac{1}{d !} \sum_{\sigma \in \mathcal{P}_{d}} \int \max _{S_{d}}(\mid \leq i \leq d)\left(x_{\sigma(i)} \mid t_{i}\right) P_{\tilde{\mathbf{Z}}}(d \boldsymbol{t}) \\
& =\int_{S_{d}} \frac{1}{d !} \sum_{\sigma \in \mathcal{P}_{d}} \max _{1 \leq i \leq d}\left(\left|x_{\sigma(i)}\right| t_{i}\right) P_{\tilde{\mathbf{Z}}}(d \boldsymbol{t}) \\
& =\int_{S_{d}}\|\boldsymbol{x}\|_{D_{t}} P_{\tilde{\mathbf{Z}}}(d \boldsymbol{t})=E\left(\|\boldsymbol{x}\|_{D_{\tilde{Z}}}\right) .
\end{aligned}
$$

Acknowledgments The authors are indebted to three anonymous reviewers and an Associate Editor for their careful reading of the manuscript. This paper has benefited a lot from their extensive and constructive remarks.

Funding Open Access funding enabled and organized by Projekt DEAL.

Open Access This article is licensed under a Creative Commons Attribution 4.0 International License, which permits use, sharing, adaptation, distribution and reproduction in any medium or format, as long as you give appropriate credit to the original author(s) and the source, provide a link to the Creative Commons licence, and indicate if changes were made. The images or other third party material in this article are included in the article's Creative Commons licence, unless indicated otherwise in a credit line to the material. If material is not included in the article's Creative Commons licence and your intended use is not permitted by statutory regulation or exceeds the permitted use, you will need to obtain permission directly from the copyright holder. To view a copy of this licence, visit http://creativecommons.org/licenses/by/4.0/.

\section{References}

Balkema, A.A., Resnick, S.I.: Max-infinite divisibility. J. Appl. Probab. 14(2), 309-319 (1977). https://doi.org/10.2307/3213001

Barbe, P., Fougères, A.L., Genest, C.: On the tail behavior of sums of dependent risks. Astin Bull. 36(2), 361-373 (2006). https://doi.org/10.1017/S0515036100014550

Cooley, D., Thibaud, E.: Decompositions of dependence for high-dimensional extremes. Biometrika 106(3), 587-604 (2019). https://doi.org/10.1093/biomet/asz028

Deheuvels, P.: Probabilistic aspects of multivariate extremes. In: Tiago de Oliveira, J. (ed.) Statistical Extremes and Applications, pp. 117-130 (1984). D. Reidel, Dordrecht, https://doi.org/10.1007/ 978-94-017-3069-3_9

de Haan, L., Resnick, S.: Limit theory for multivariate sample extremes. Probab. Theor. Relat. Fields 40(4), 317-337 (1977). https://doi.org/10.1007/BF00533086

Falk, M.: It was 30 years ago today when Laurens de Haan went the multivariate way. Extremes 11(1), 55-80 (2008). https://doi.org/10.1007/s10687-007-0045-z

Falk, M.: Multivariate extreme value theory and D-Norms. Springer International, New York (2019). https://doi.org/10.1007/978-3-030-03819-9 
Falk, M., Hüsler, J., Reiss, R.D. Laws of Small Numbers: Extremes and Rare Events, 2nd edn. Basel, Birkhäuser (2004). https://doi.org/10.1007/978-3-0348-7791-6

Falk, M., Hüsler, J., Reiss, R.D. Laws of Small Numbers: Extremes and Rare Events, 3rd edn. Basel, Birkhäuser (2011). https://doi.org/10.1007/978-3-0348-0009-9

Galambos, J. The Asymptotic Theory of Extreme Order Statistics, 2nd edn. Krieger, Malabar (1987)

Jessen, A.H., Mikosch, T.: Regularly varying functions. Publications de l'Institut Mathematique. Nouvelle Sé,rie 80, 171-192 (2006). https://doi.org/10.2298/PIM0694171H

Larsson, M., Resnick, S.I.: Extremal dependence measure and extremogram: The regulary varying case. Extremes 15(2), 231-256 (2012). https://doi.org/10.1007/s10687-011-0135-9

Mai, J.F., Scherer, M.: On the structure of exchangeable extreme-value copulas. J. Multivar. Anal. 180, 104670 (2020). https://doi.org/10.1016/j.jmva.2020.104670

Molchanov, I.: Convex geometry of max-stable distributions. Extremes 11(3), 235-259 (2008). https://doi.org/10.1007/s10687-008-0055-5

Nelsen, R.B. An Introduction to Copulas, 2nd edn. Springer Series in Statistics, Springer, New York (2006). https://doi.org/10.1007/0-387-28678-0

Ng, K.W., Tian, G.L., Tang, M.L.: Dirichlet and related distributions. theory, Methods and Applications. Wiley Series in Probability and Statistics. Wiley, Chichester (2011). https://doi.org/10.1002/9781119995784

Pickands, J. III.: Multivariate extreme value distributions. Proc. 43th Session ISI (Buenos Aires) 859-878 (1981)

Reiss, R.D.: A Course on Point Processes. Springer, New York (1993). https://doi.org/10.1007/978-1-46 13-9308-5

Resnick, S.I.: Extreme Values, Regular Variation, and Point Processes. Springer Series in Operations Research and Financial Engineering. Springer, New York (2008)

Ressel, P.: Homogeneous distributions - and a spectral representation of classical mean values and stable tail dependence functions. J. Multivariate Anal. 117, 246-256 (2013). https://doi.org/10.1016/j. jmva.2013.02.013

Rootzén, H., Segers, J., Wadsworth, J.L.: Multivariate peaks over thresholds models. Extremes 21, 115145 (2018). https://doi.org/10.1007/s10687-017-0294-4

Sklar, A.: Fonctions de répartition à $n$ dimensions et leurs marges. Pub. Inst. Stat. Univ. Paris 8, 229-231 (1959)

Sklar, A.: Random Variables, Distribution Functions, and Copulas - a Personal Look Backward and Forward. In: Rüschendorf, L., Schweizer, B., Taylor, M.D. (eds.) Distributions with Fixed Marginals and Related Topics, Lecture Notes - Monograph Series, vol. 28, pp. 1-14. Institute of Mathematical Statistics, Hayward, CA (1996). https://doi.org/10.1214/lnms/1215452606

Takahashi, R.: Some properties of multivariate extreme value distributions and multivariate tail equivalence. Ann. Inst. Stat. Math. 39(1), 637-647 (1987). https://doi.org/10.1007/BF02491496

Takahashi, R.: Characterizations of a multivariate extreme value distribution. Adv. Appl. Probab. 20(1), 235-236 (1988). https://doi.org/10.2307/1427279

Vatan, P.: Max-infinite divisibility and max-stability in infinite dimensions. In: Beck, A., Dudley, R., Hahn, M., Kuelbs, J., Marcus, M. (eds.) Probability in Banach Spaces V: Proceedings of the International Conference held in Medford, USA, July 16, 1984, Lecture Notes in Mathematics, vol. 1153, pp. 400425. Springer, Berlin (1985). https://doi.org/10.1007/BFb0074963

Publisher's note Springer Nature remains neutral with regard to jurisdictional claims in published maps and institutional affiliations. 